\title{
Synthesis of forsythenethoside A, a neuroprotective macrocyclic phenylethanoid glycoside, and NMR analysis of conformers
}

Zhifei Hu, ${ }^{\dagger, *}$ Alba Silipo, ${ }^{* \S}$ Wei Li, ${ }^{\ddagger}, \mathbf{I I}$ Antonio Molinaro, ${ }^{\S}$ Biao Yu**

†School of Physical Science and Technology, ShanghaiTech University, 393 Huaxia Middle Road, Shanghai 201210, China.

${ }^{\ddagger}$ State Key Laboratory of Bioorganic and Natural Products Chemistry, Center for Excellence in Molecular Synthesis, Shanghai Institute of Organic Chemistry, University of Chinese Academy of Sciences, Chinese Academy of Sciences, 345 Lingling Road, Shanghai 200032, China

${ }^{\S}$ Department of Chemical Sciences, University of Naples "Federico II", Via Cintia 4, 80126 Napoli, Italy

IIDepartment of Medicinal Chemistry, China Pharmaceutical University, 639 Longmian Avenue, Nanjing, Jiangsu 211198, China.

\section{Table of Contents}

HSQC/ROESY spectra, NOE derived distances, and conformation of $\mathbf{1 5}$............S2

ROESY spectra and conformation of forsythenethoside A (1) ..................S4

${ }^{1} \mathrm{H}$ and ${ }^{13} \mathrm{C}\{1 \mathrm{H}\}$ NMR data for synthetic and natural forsythenethoside A (1)........S6

NOE derived distances of forsythenethoside A (1) ............................ 7

NMR spectra of compounds $1-4,8-11,13-17$, and $19-22 \ldots \ldots \ldots \ldots \ldots \ldots \ldots . . . . . \ldots$

MD simulation report for forsythenethoside A (1) and 15 .....................S42 


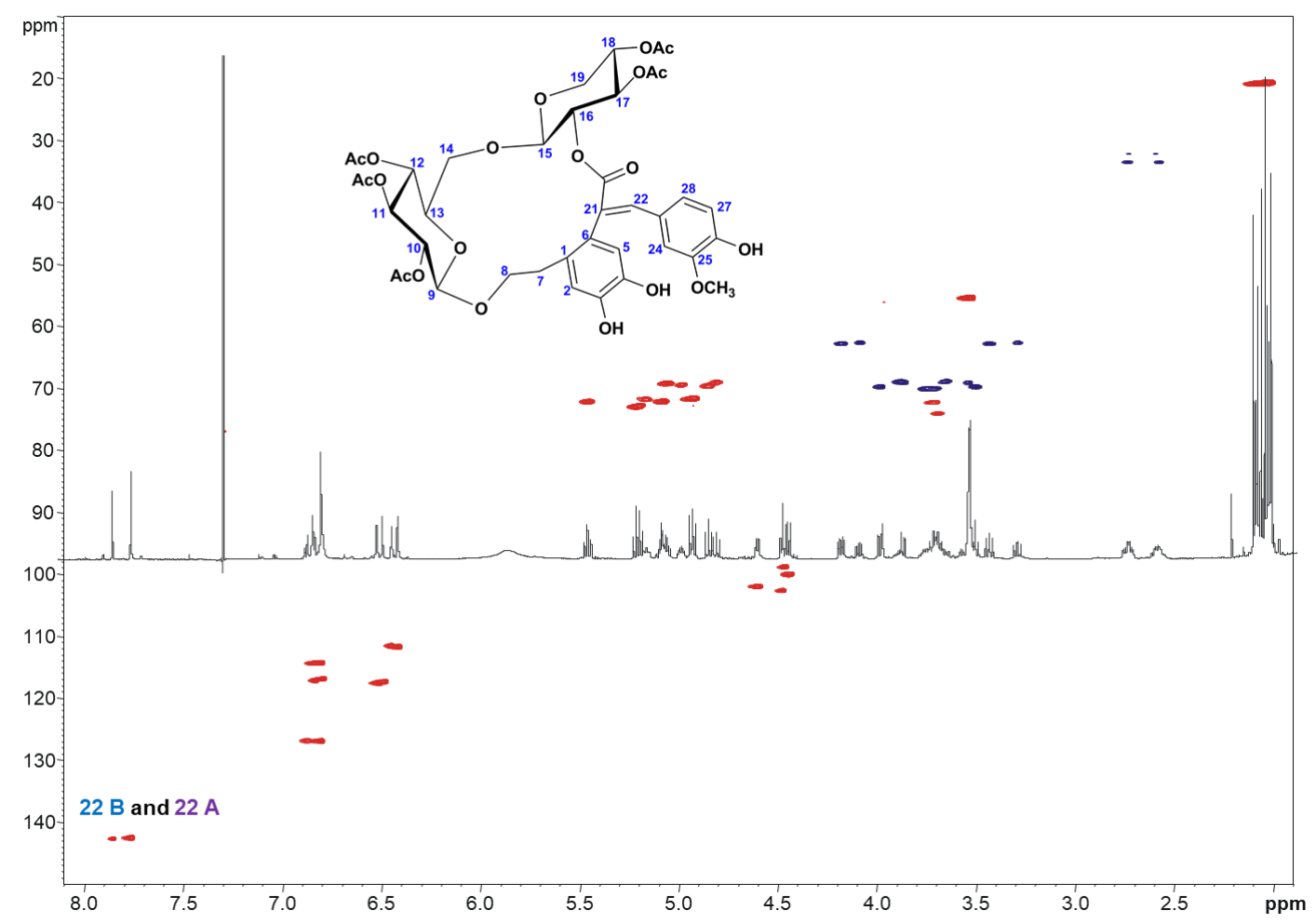

Figure S1 HSQC NMR spectrum of macrocycle 15 in $\mathrm{CDCl}_{3}$

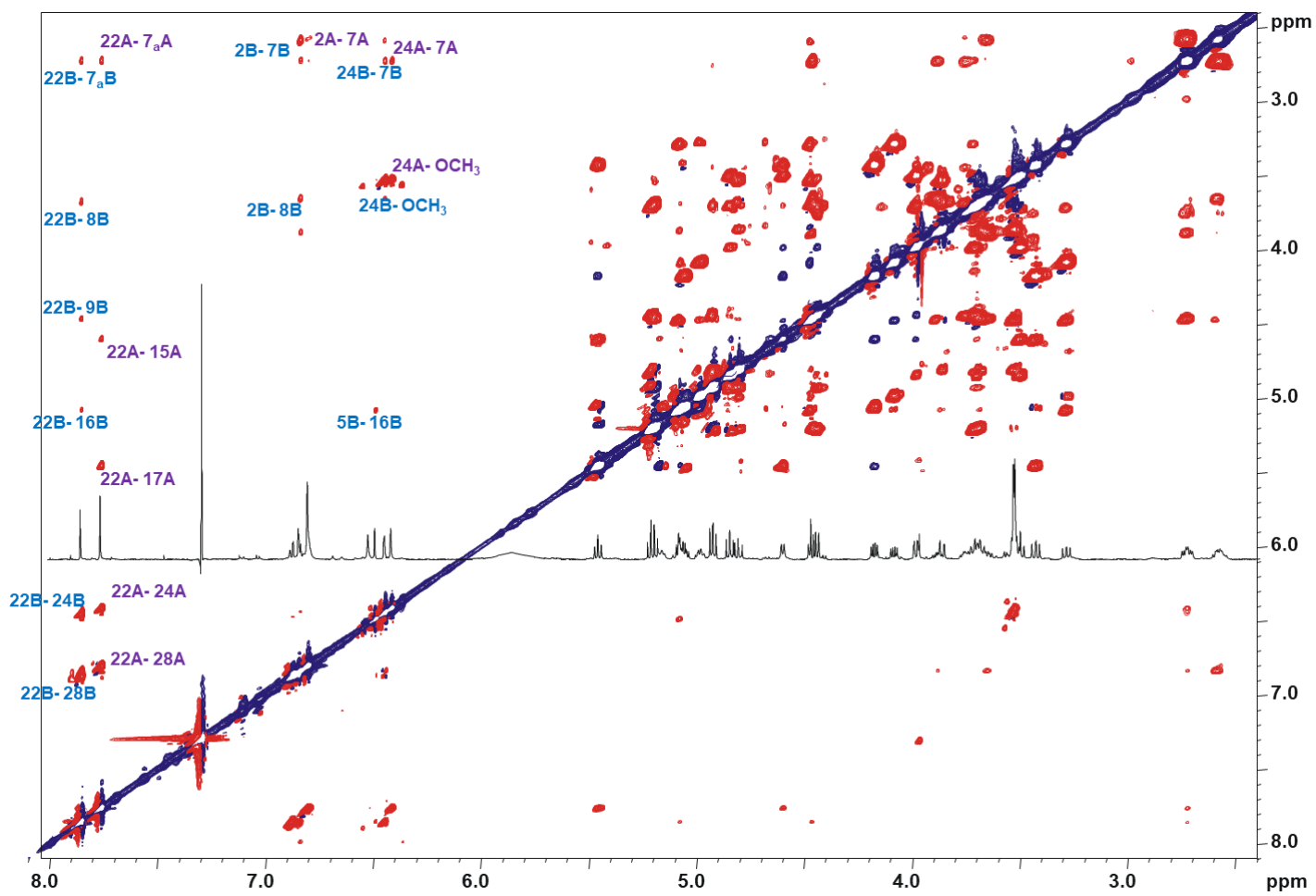

Figure S2 ROESY NMR spectrum of macrocycle 15 in $\mathrm{CDCl}_{3}$ 
Table S1: Macrocycle 15, NOE derived distances of $\mathbf{A}$ and $\mathbf{B}$ conformers

\begin{tabular}{|c|c|c|c|}
\hline \multicolumn{5}{|c|}{ NOE- derived distances (in Å) } \\
\hline A conformer & & B conformer & \\
\hline $22-17$ & 2.886774042 & $22-16$ & 4.208398042 \\
\hline $22-15$ & 3.508213014 & $22-9$ & 3.863252547 \\
\hline $22-7 a$ & 3.396990525 & $2-7 a$ & 2.957050927 \\
\hline $22-7 b$ & 3.459021539 & $2-7 b$ & 3.638711971 \\
\hline & & $5-16$ & 3.583244788 \\
\hline & & $2-8 a$ & 3.361360575 \\
\hline $22-28$ overlapping with & & $2-8 b$ & 3.600798077 \\
\hline $22-24$ & 2.054210236 & $22-28$ overlapping with & \\
\hline & 2.5 & $22-24$ & 2.075377677 \\
\hline
\end{tabular}

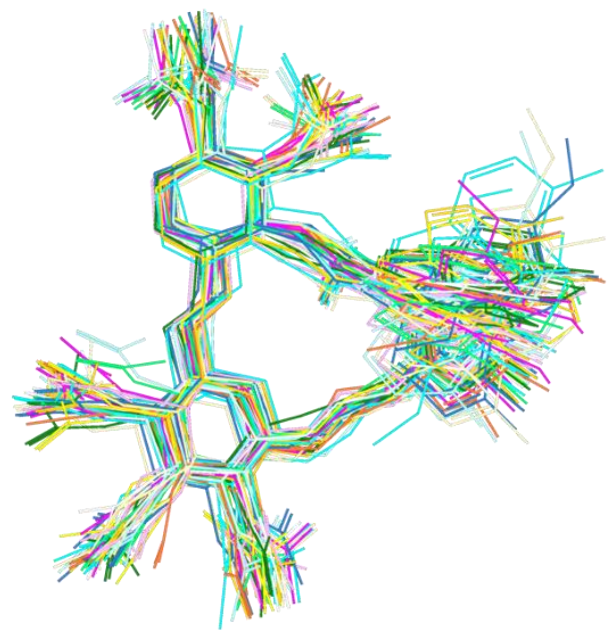

A conformers

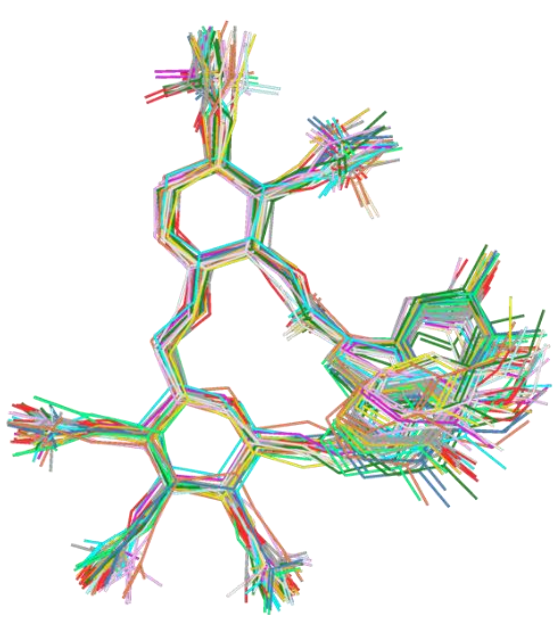

B conformers

Figure S3 The two main conformational families of macrocycle 15: A and B conformers 


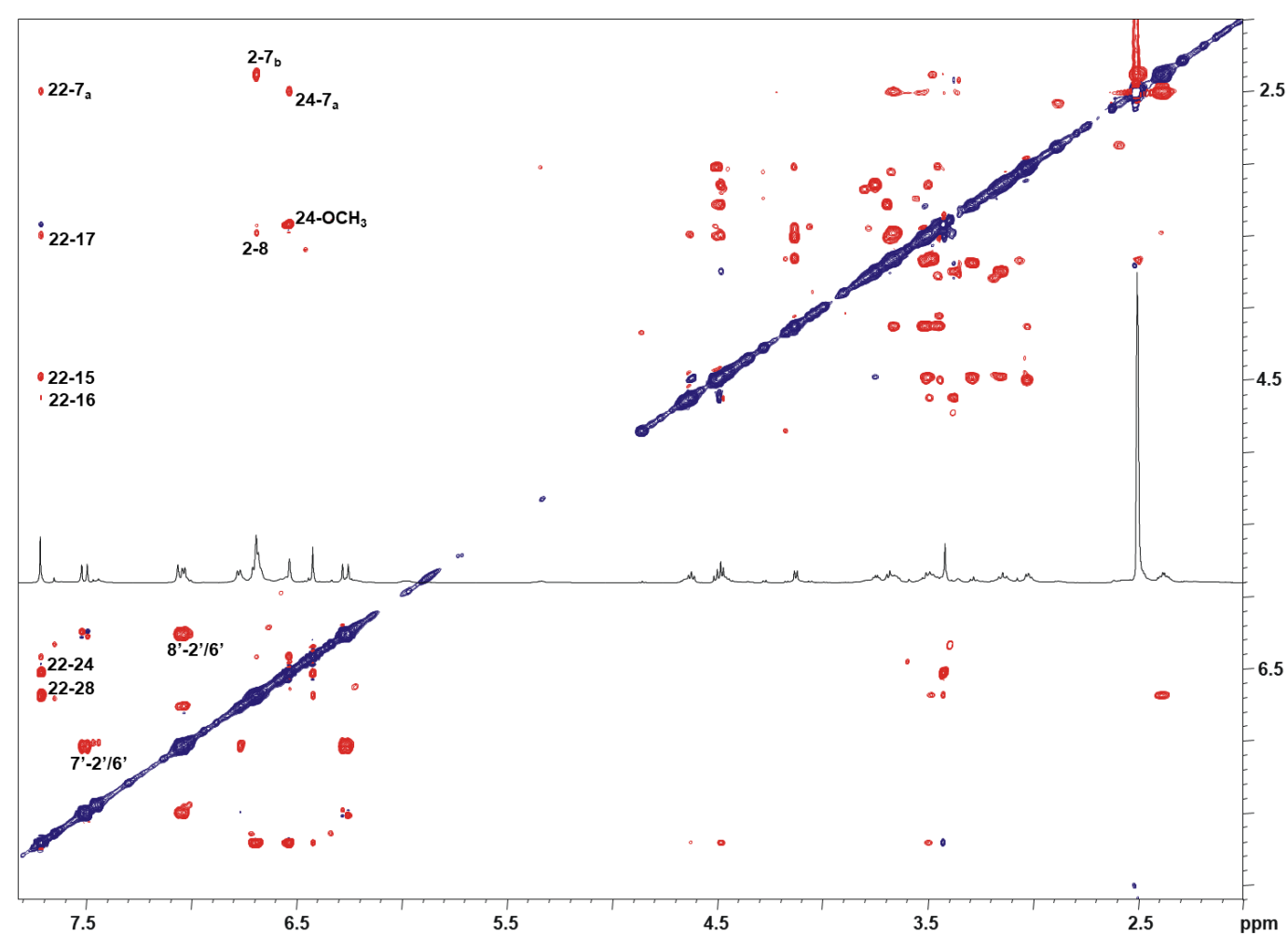

Figure S4 ROESY NMR spectrum of forsythenethoside A (1) in DMSO-d6

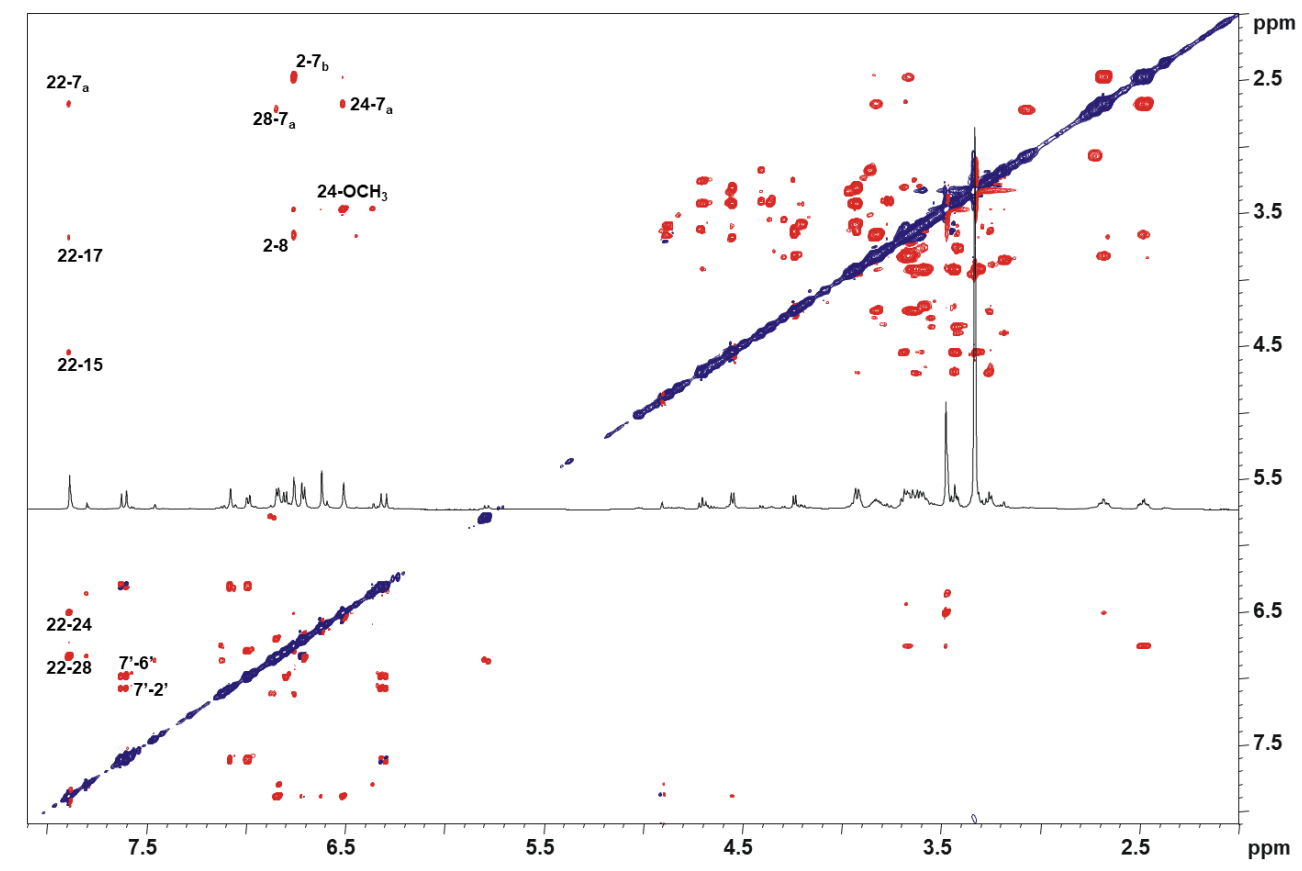

Figure S5 ROESY NMR spectrum of forsythenethoside A (1) in $\mathrm{CD}_{3} \mathrm{OD}$ 


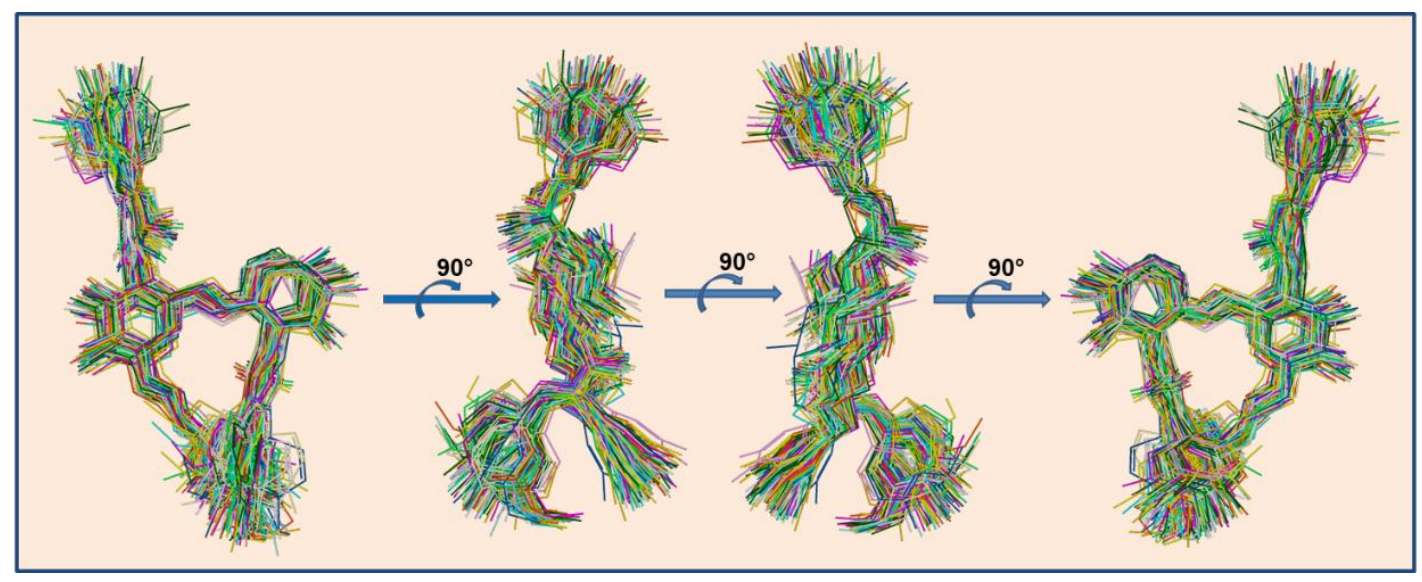

Figure S6 View of representative conformers of forsythenethoside A (1).
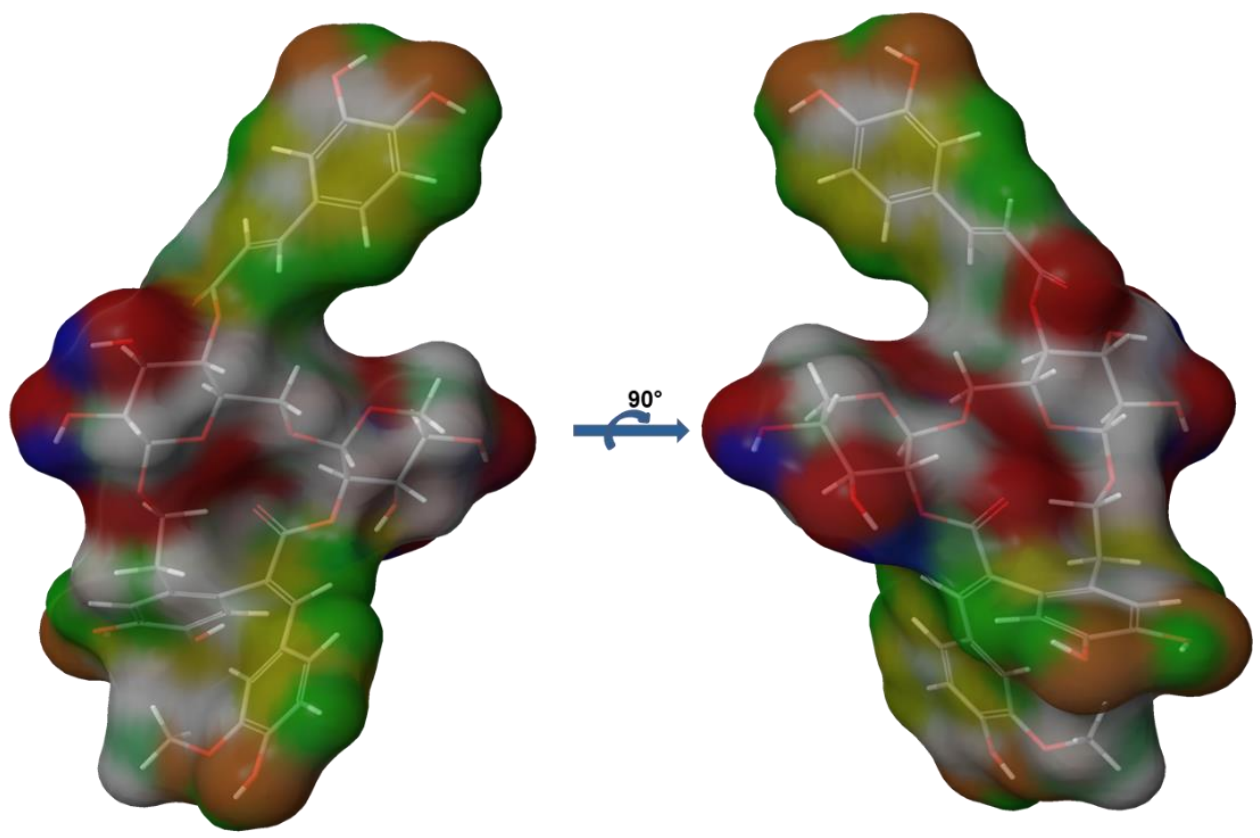

Figure S7 View of a representative conformer with Connelly surface of forsythenethoside A (1). 
Table S2 The ${ }^{1} \mathrm{H}$ and ${ }^{13} \mathrm{C}$ NMR data for synthetic and natural forsythenethoside A (1).

\begin{tabular}{|c|c|c|c|c|}
\hline \multirow[b]{2}{*}{ position } & \multicolumn{2}{|c|}{${ }^{1} \mathrm{H}$ NMR data/ppm (Hz) } & \multicolumn{2}{|c|}{${ }^{13} \mathrm{C}$ NMR data/ppm } \\
\hline & natural & synthetic & natural & synthetic \\
\hline 1 & & & 127.0 & 127.0 \\
\hline 2 & $6.67, \mathrm{~s}$ & $6.67, \mathrm{~s}$ & 117.2 & 117.2 \\
\hline 3 & & & 144.4 & 144.3 \\
\hline 4 & & & 145.0 & 144.9 \\
\hline 5 & $6.40, \mathrm{~s}$ & $6.40, \mathrm{~s}$ & 117.2 & 117.2 \\
\hline 6 & & & 127.0 & 127.1 \\
\hline $7 \mathrm{a}$ & $2.48, \mathrm{~m}$ & $2.48, \mathrm{~m}$ & & \\
\hline $7 b$ & 2.37, $\mathrm{m}$ & $2.38, \mathrm{~m}$ & 33.4 & 33.4 \\
\hline $8 a$ & $3.64, \mathrm{~m}$ & $3.64, \mathrm{~m}$ & 600 & 601 \\
\hline $8 b$ & $3.45, \mathrm{~m}$ & $3.45, \mathrm{~m}$ & 09.0 & 09.1 \\
\hline 9 & $4.10, \mathrm{~d}(8.0)$ & 4.11, d (7.9) & 102.0 & 102.0 \\
\hline 10 & $3.01, \mathrm{t}(8.0)$ & $3.02, \mathrm{~m}$ & 73.5 & 73.5 \\
\hline 11 & $3.44, \mathrm{~m}$ & $3.44, \mathrm{~m}$ & 73.9 & 73.9 \\
\hline 12 & $4.48, \mathrm{t}(9.5)$ & $4.48, \mathrm{t}(9.5)$ & 71.7 & 71.7 \\
\hline 13 & $3.50, \mathrm{~m}$ & $3.51, \mathrm{~m}$ & 71.9 & 71.9 \\
\hline $14 \mathrm{a}$ & $3.67, \mathrm{~m}$ & $3.67, \mathrm{~m}$ & 603 & 603 \\
\hline $14 b$ & $3.27, \mathrm{~m}$ & $3.27, \mathrm{~m}$ & 09.3 & 09.3 \\
\hline 15 & $4.47, \mathrm{~d}(7.5)$ & $4.47, \mathrm{~d}(7.5)$ & 101.6 & 101.6 \\
\hline 16 & $4.61, \mathrm{t}(7.5)$ & $4.61, \mathrm{t}(7.5)$ & 74.0 & 74.0 \\
\hline 17 & $3.48, \mathrm{~m}$ & $3.48, \mathrm{~m}$ & 74.2 & 74.2 \\
\hline 18 & $3.36, \mathrm{~m}$ & $3.37, \mathrm{~m}$ & 69.8 & 69.8 \\
\hline $19 \mathrm{a}$ & $3.71, \mathrm{~m}$ & $3.73, \mathrm{dd}(11.1,5.1)$ & & \\
\hline $19 b$ & $3.13, \mathrm{t}(11.0)$ & $3.13, \mathrm{t}(10.9)$ & 65.6 & 65.6 \\
\hline 20 & & & 166.0 & 166.0 \\
\hline 21 & & & 126.5 & 126.5 \\
\hline 22 & $7.68, \mathrm{~s}$ & $7.69, \mathrm{~s}$ & 139.4 & 139.4 \\
\hline 23 & & & 125.8 & 125.8 \\
\hline 24 & 6.51, brs & $6.52, \mathrm{~d}(1.2)$ & 112.9 & 113.0 \\
\hline 25 & & & 147.1 & 147.1 \\
\hline 26 & & & 148.4 & 148.3 \\
\hline 27 & $6.65, \mathrm{~d}(8.5)$ & $6.66, \mathrm{~m}$ & 115.3 & 115.3 \\
\hline 28 & 6.68, brd $(8.5)$ & $6.67, \mathrm{~m}$ & 125.2 & 125.2 \\
\hline $25-\mathrm{OCH}_{3}$ & $3.40, \mathrm{~s}$ & $3.41, \mathrm{~s}$ & 54.8 & 54.8 \\
\hline $1^{\prime}$ & & & 125.3 & 125.4 \\
\hline $2^{\prime}$ & 7.04, brs & 7.04, brs & 115.0 & 115.0 \\
\hline $3^{\prime}$ & & & 145.6 & 145.6 \\
\hline $4^{\prime}$ & & & 148.7 & 148.6 \\
\hline $5^{\prime}$ & $6.74, \mathrm{~d}(8.0)$ & $6.76, \mathrm{~d}(8.1)$ & 115.8 & 115.8 \\
\hline $6^{\prime}$ & 7.01, brd $(8.0)$ & $7.01, \mathrm{dd}(8.2,1.7)$ & 121.5 & 121.5 \\
\hline $7^{\prime}$ & $7.48, \mathrm{~d}(15.5)$ & $7.49, \mathrm{~d}(15.8)$ & 146.0 & 146.0 \\
\hline $8^{\prime}$ & $6.23, \mathrm{~d}(15.5)$ & $6.24, \mathrm{~d}(15.9)$ & 113.4 & 113.5 \\
\hline $9^{\prime}$ & & & 166.2 & 166.1 \\
\hline
\end{tabular}


Table S3 Key NOE derived distances of the major conformer for forsythenethoside A (1) in DMSO- $d 6$ and $\mathrm{CD}_{3} \mathrm{OD}$.

\begin{tabular}{|c|c|c|}
\hline \multicolumn{2}{|c|}{ NOE- derived distances (in $\AA$ ) } \\
\hline $\begin{array}{c}\text { Proton } \\
\text { residues }\end{array}$ & DMSO-d6 & CD3OD \\
\hline $\mathbf{2 2 - 2 4}$ & 2.71 & 2.98 \\
\hline $\mathbf{2 2 - 1 5}$ & 3.56 & 3.41 \\
\hline $\mathbf{2 2 - 1 7}$ & 3.64 & 3.32 \\
\hline $\mathbf{2 2 - 7}$ & 3.70 & 3.80 \\
\hline $\mathbf{2 2 - 2 8}$ & $2.30 *$ & 2.50 \\
\hline $\mathbf{2 4 - 5}$ & 3.28 & 3.56 \\
\hline $\mathbf{2 - 7}$ & $2.38 / \mathrm{OL}$ & $2.43 / 3.12$ \\
\hline $\mathbf{2 - 8}$ & 3.24 & 3.23 \\
\hline $\mathbf{8 - 7}$ & 3.1 & 2.97 \\
\hline $\mathbf{8 - 7}$ & 2.73 & 2.84 \\
\hline $\mathbf{2}-\mathbf{- 8}$ & 2.56 & 2.85 \\
\hline $\mathbf{2}-7$ & 2.78 & 3.05 \\
\hline $\mathbf{6}-\mathbf{9}$ & 2.51 & 2.97 \\
\hline $\mathbf{6}-7$ & 2.68 & 2.78 \\
\hline $\mathbf{9 - 8 a}$ & OL/2.75 & OL/2.85 \\
\hline $\mathbf{2 4 - 7}$ & $3.13 / 3.16$ & $3.10 /$ \\
\hline $\mathbf{2 8 - 7}$ & $3.10 \mathrm{ax}$ & $3.09 \mathrm{ax}$ \\
\hline
\end{tabular}

* distance likely overestimated due to partial overlapping with other cross peaks $\mathrm{OL}=$ not measured due to overlapping 

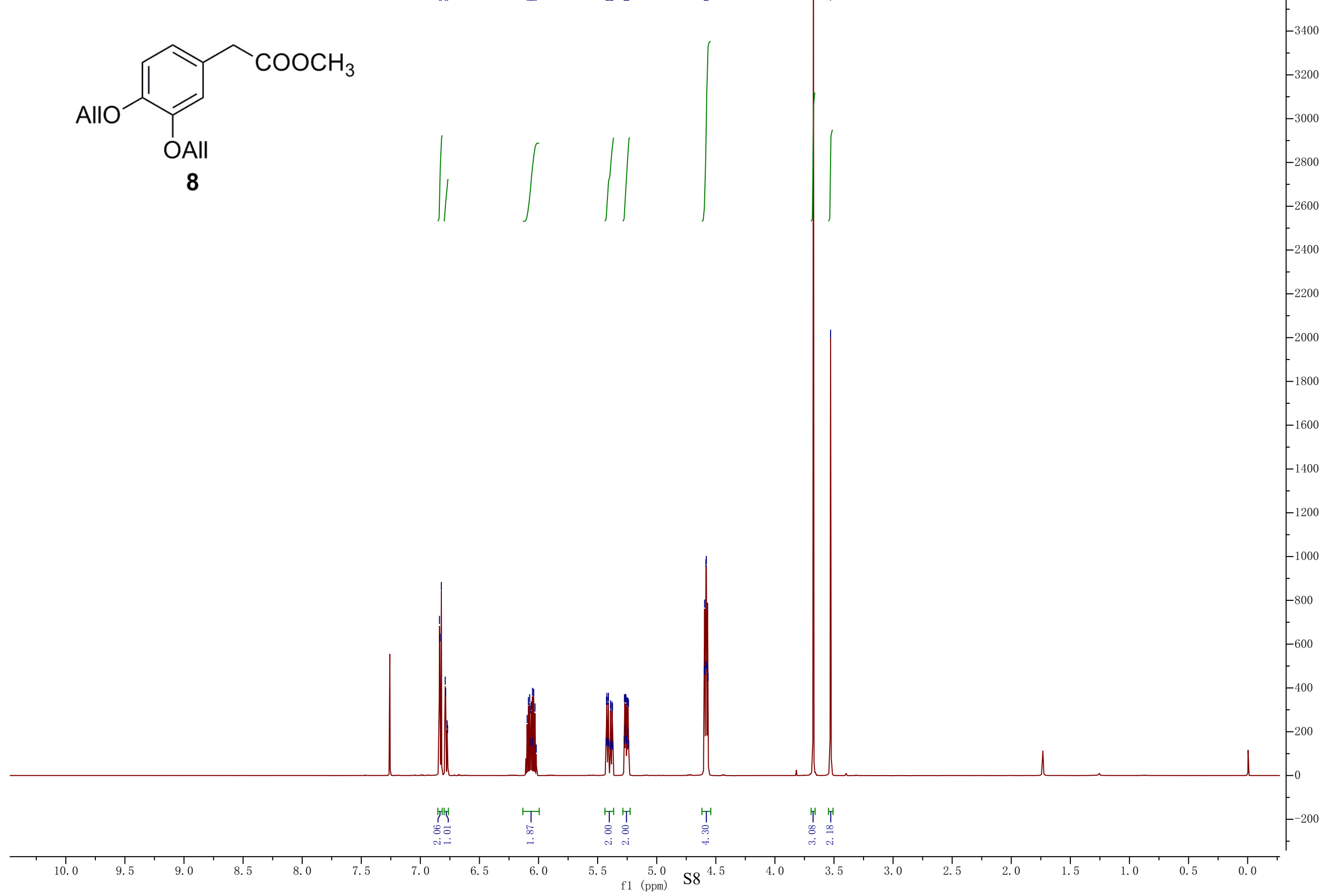
<smiles>COC(=O)Cc1ccc(O)c(O[13CH3])c1</smiles>

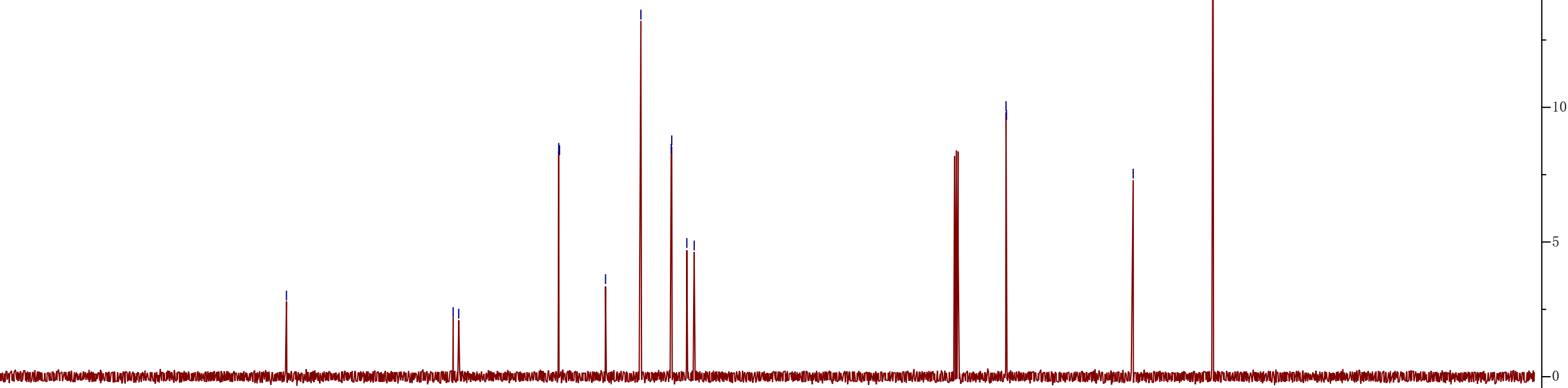


<smiles>OCCc1ccc(O)c(O)c1</smiles>
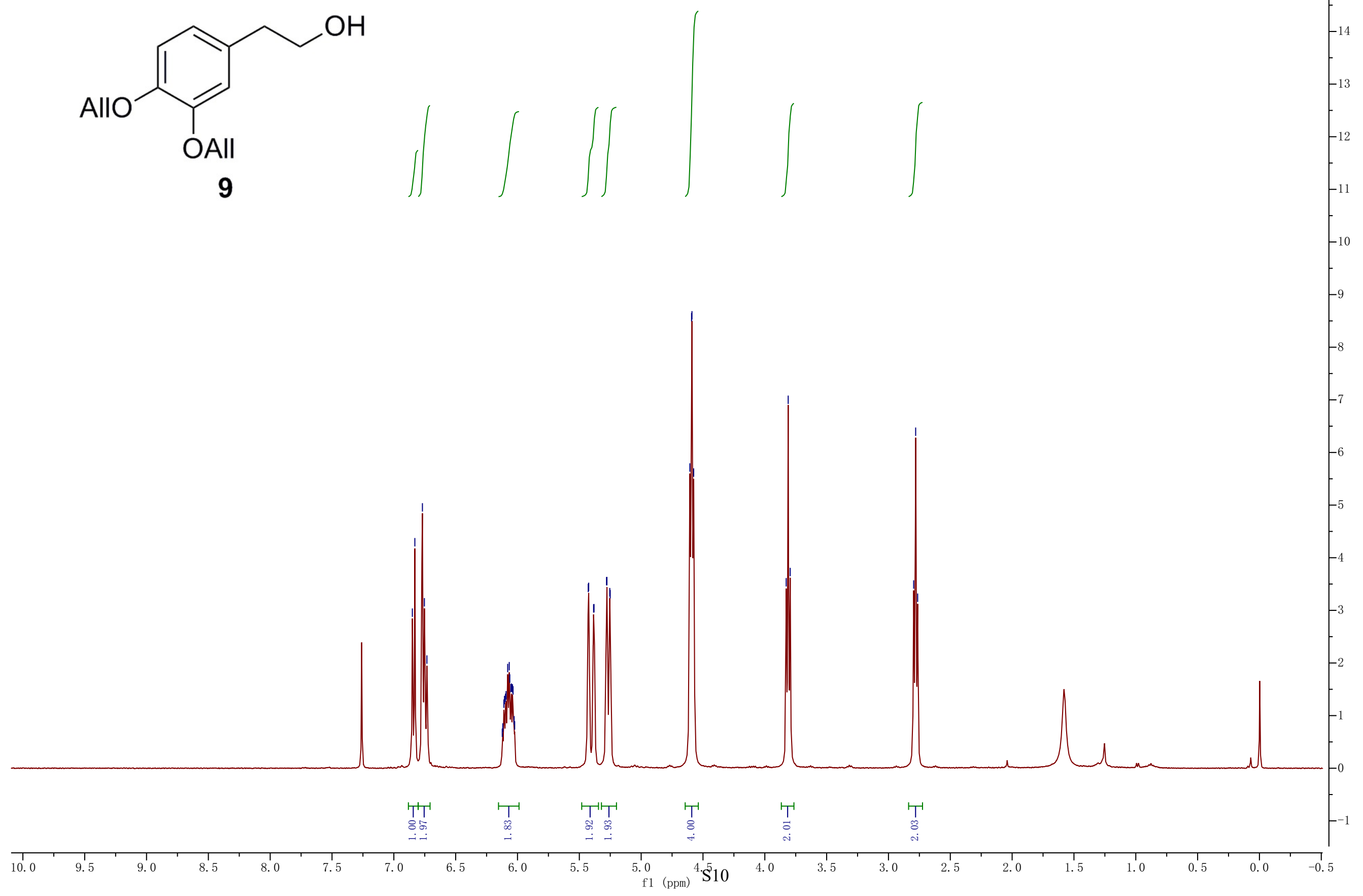

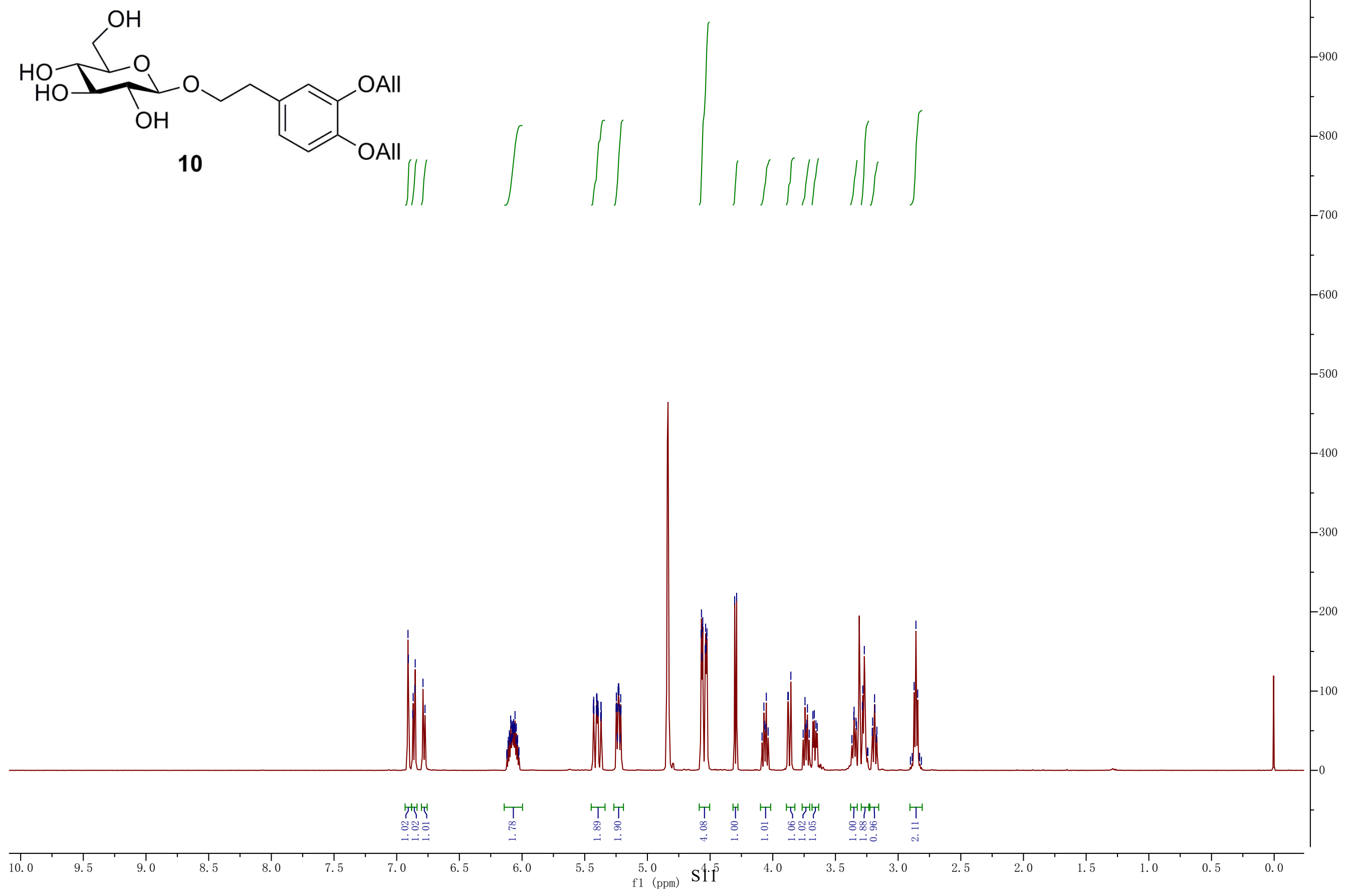
$126 \mathrm{MHz}, \mathrm{CD}_{3} \mathrm{OD}$<smiles>COc1ccc(CCOC2OC(CO)C(O)C(O)C2O)cc1OC</smiles>

10 

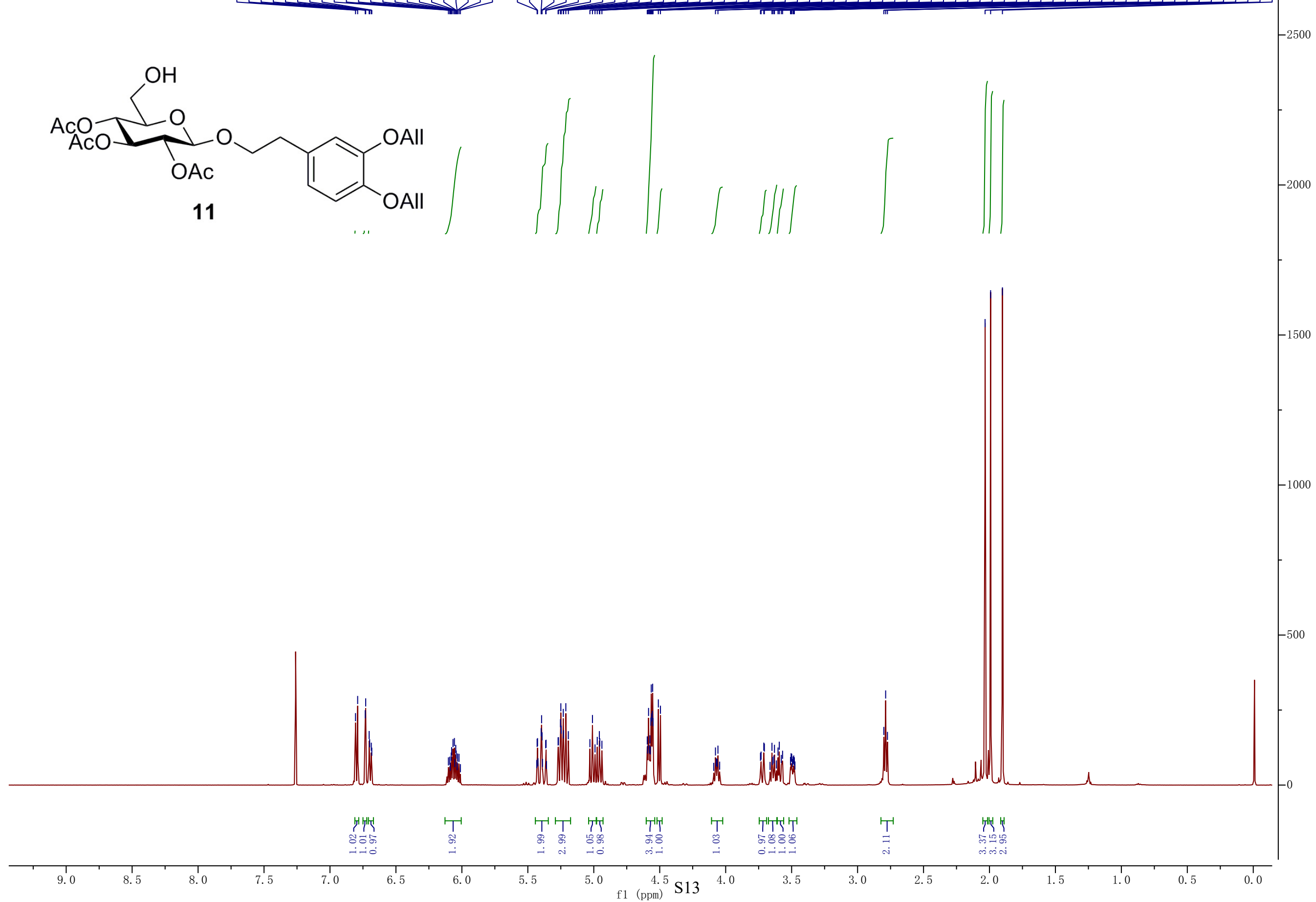

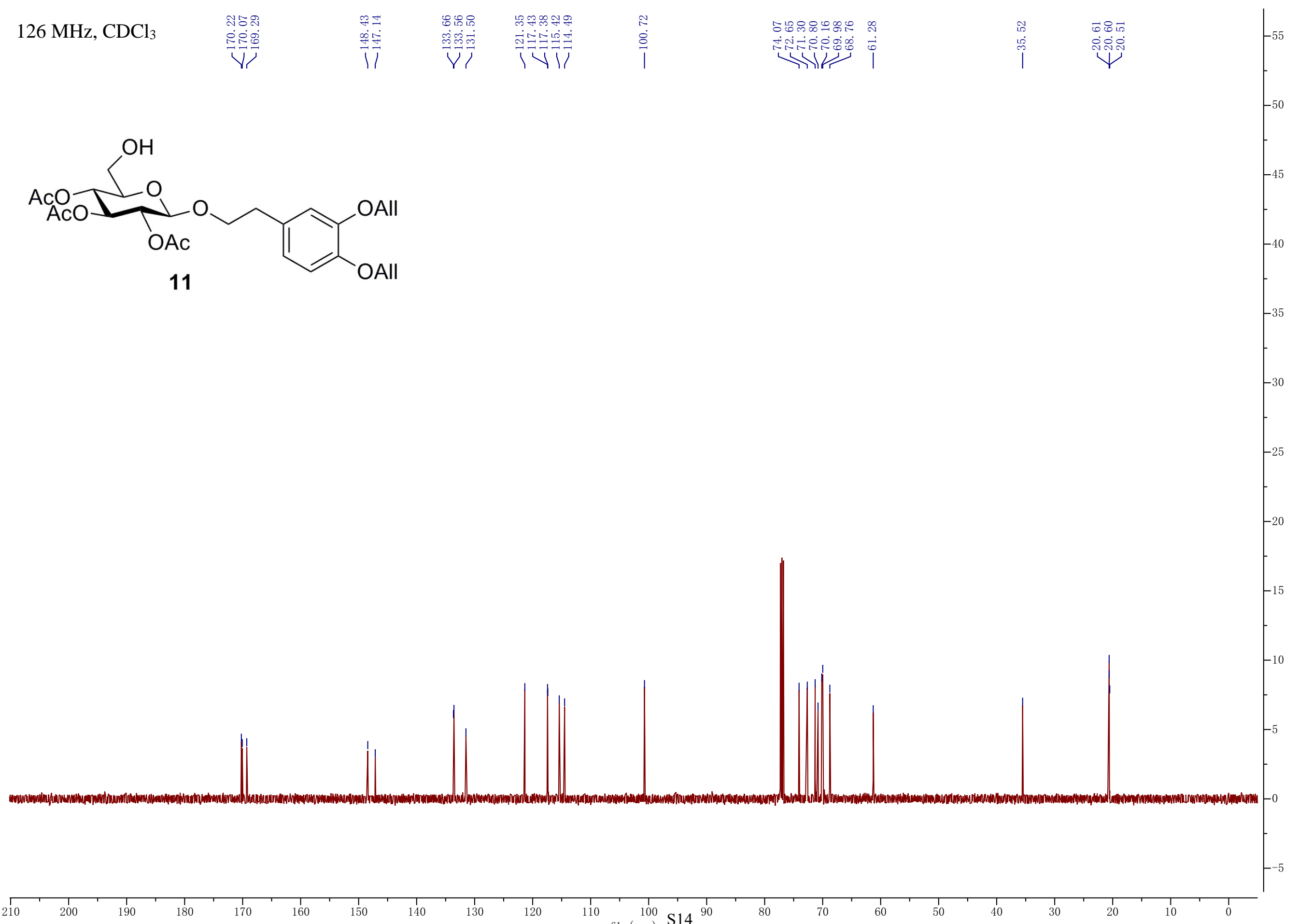


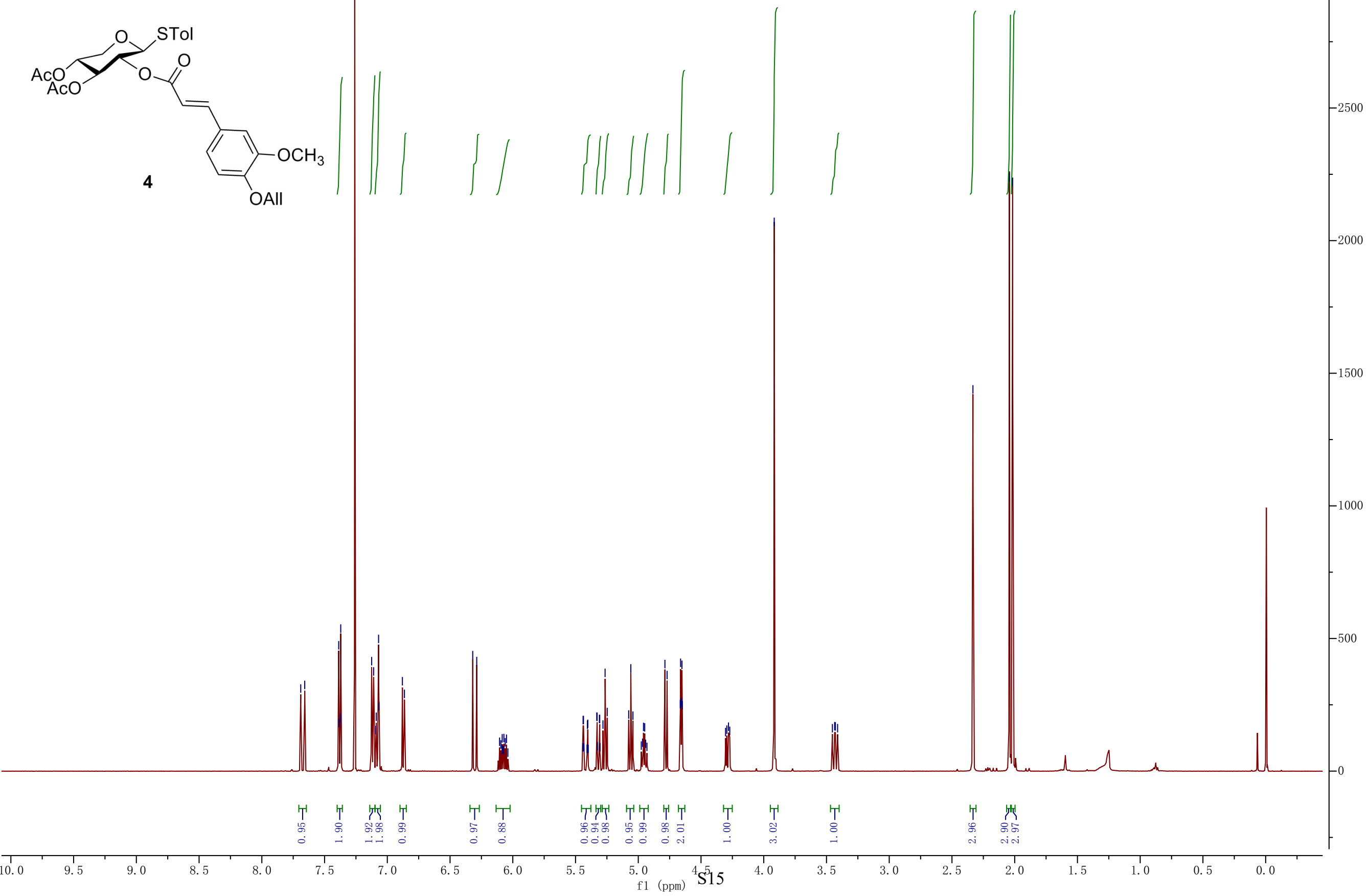


$126 \mathrm{MHz}, \mathrm{CDCl}_{3}$
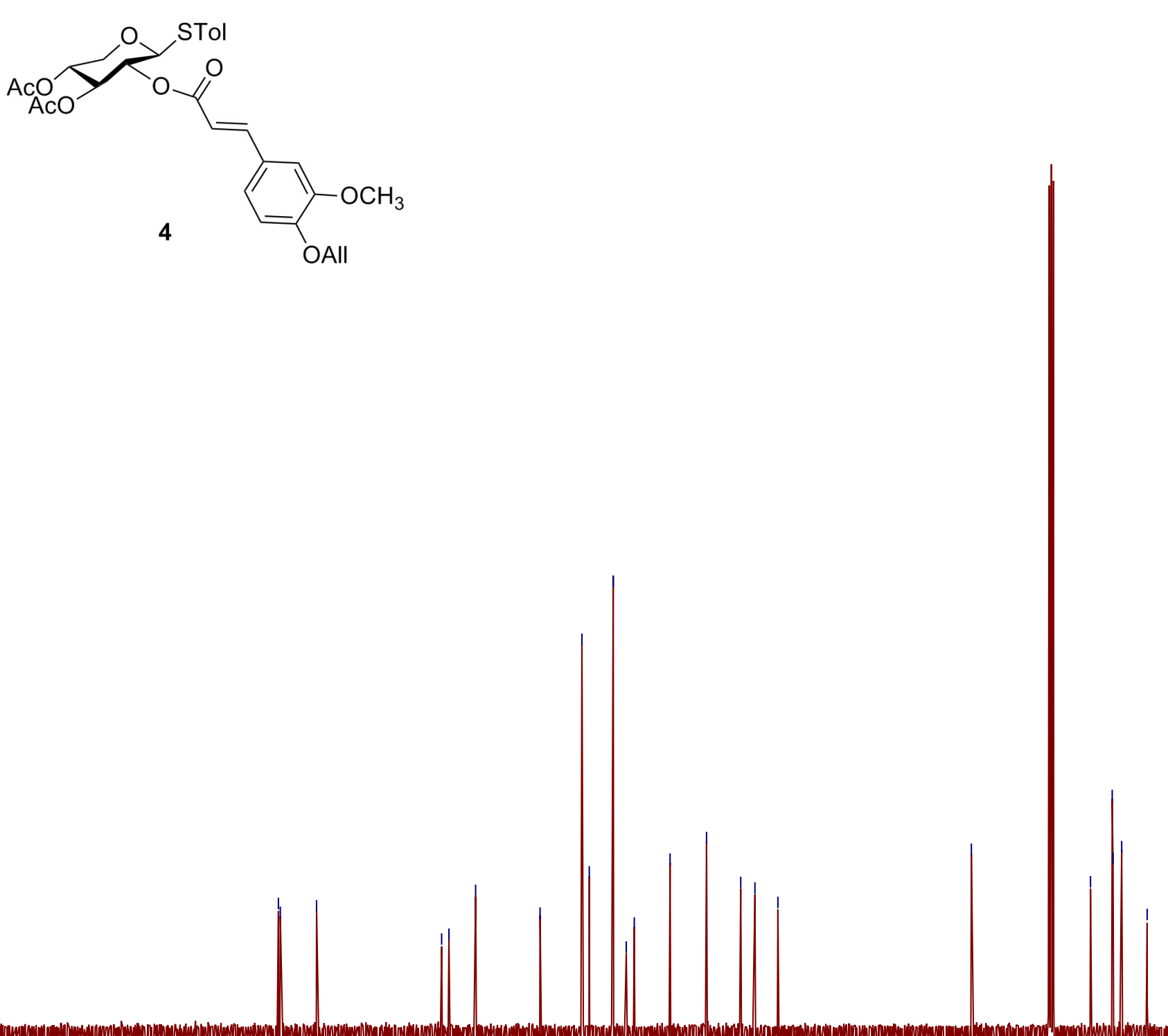

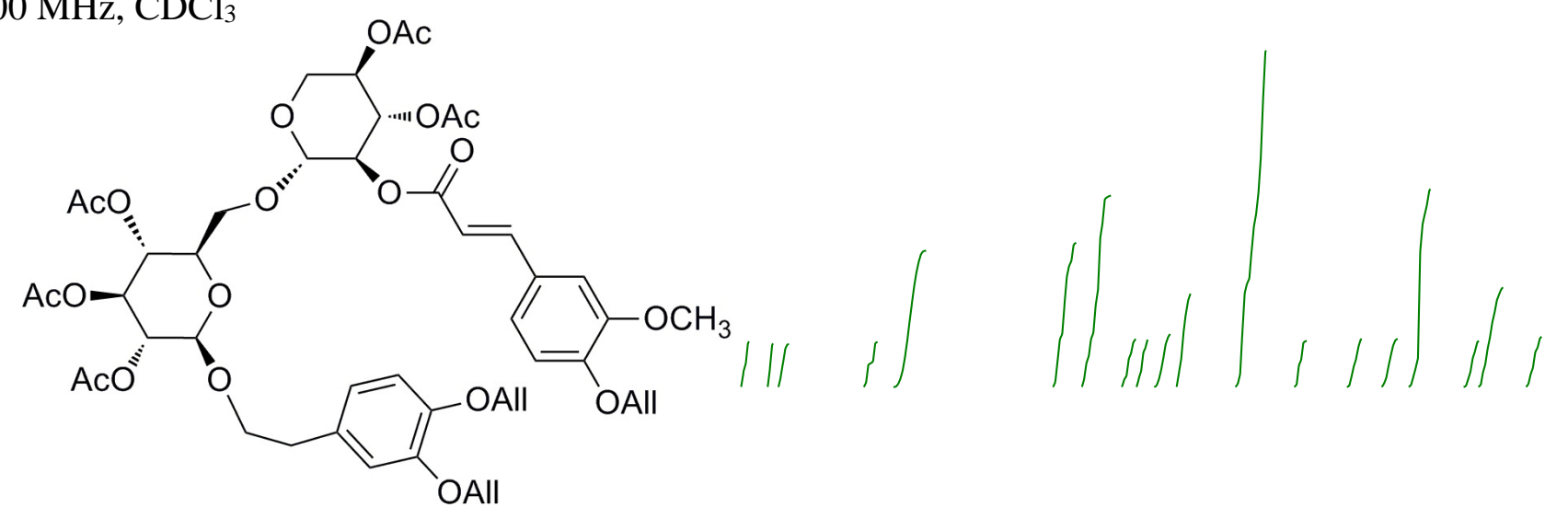

13

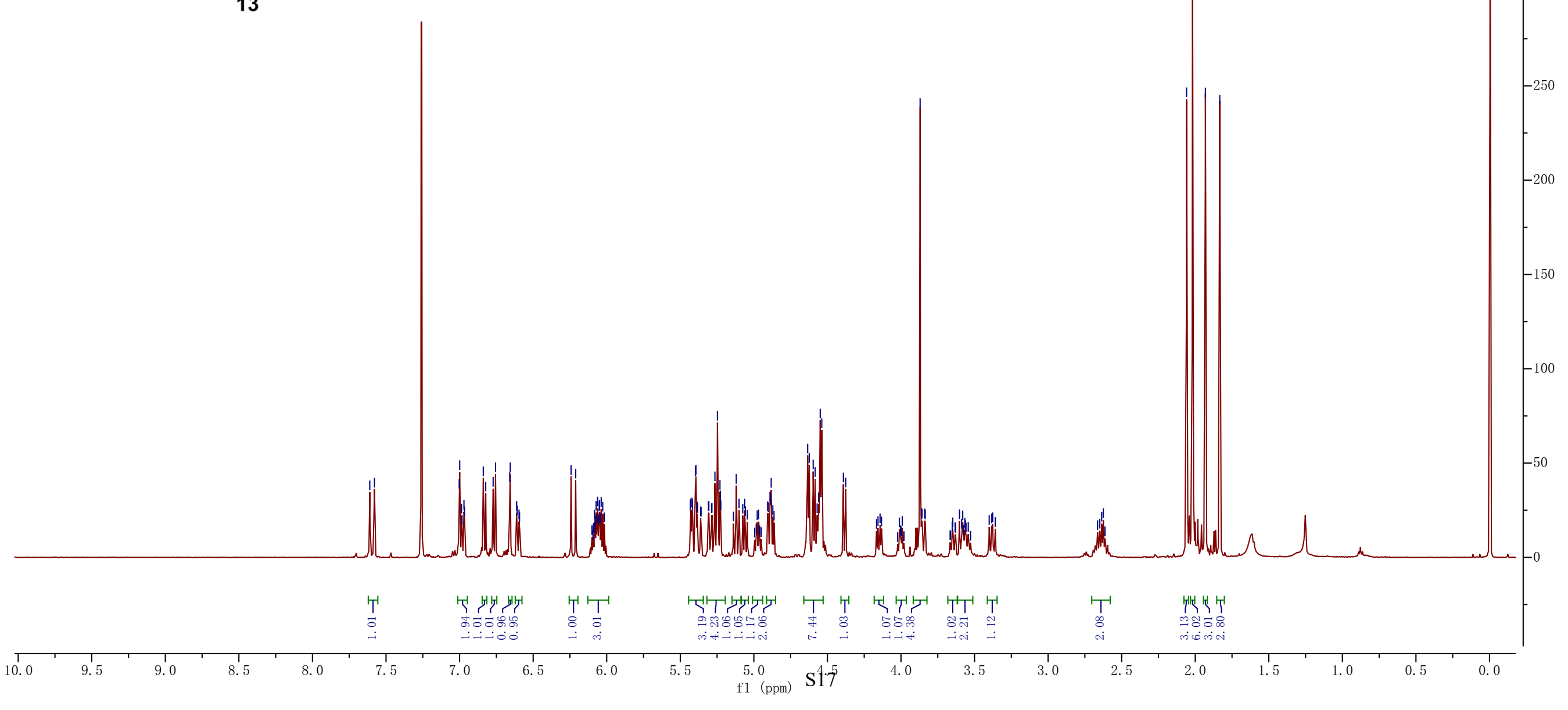


$126 \mathrm{MHz}, \mathrm{CDCl}_{3}$

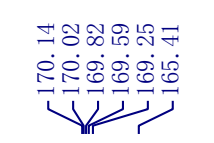

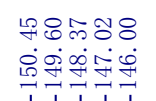

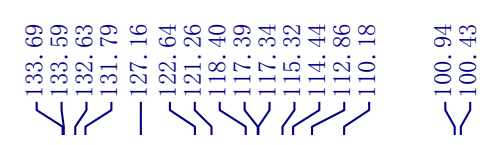

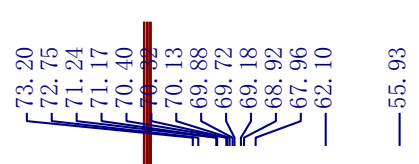

辛

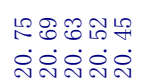

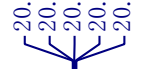

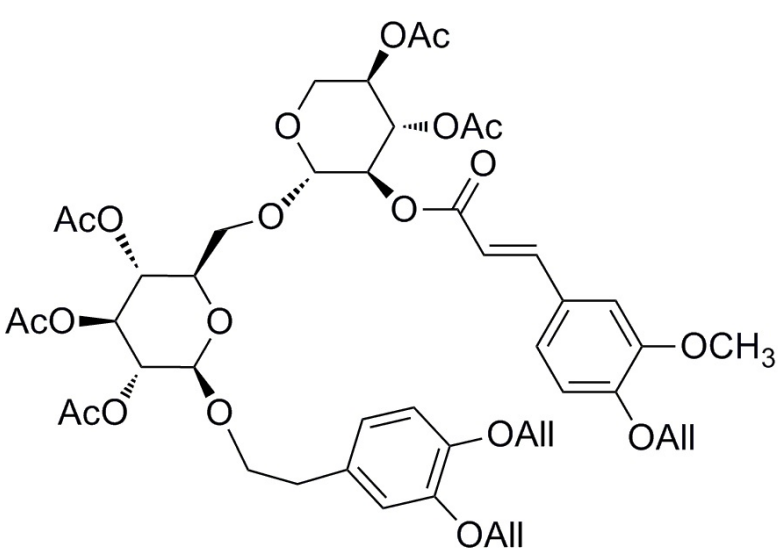

13

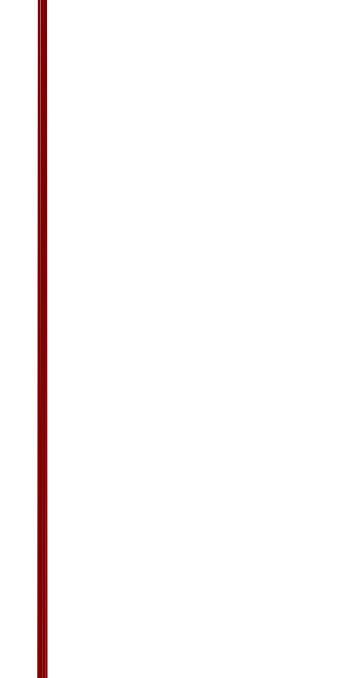

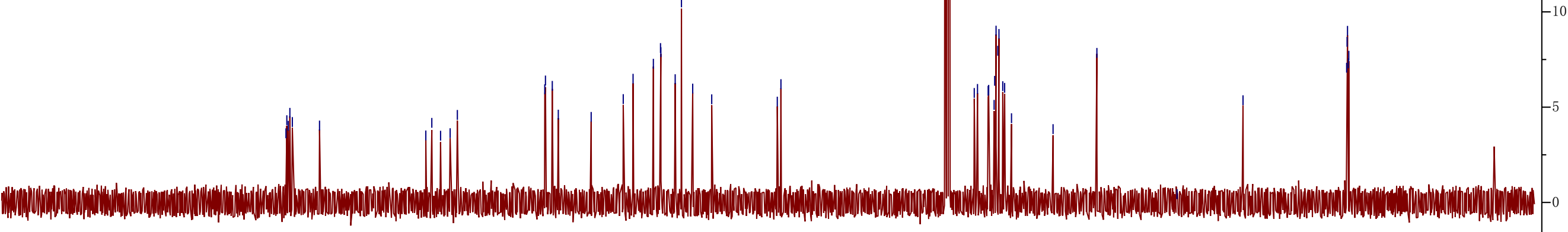


$500 \mathrm{MHz}, \mathrm{CDCl}_{3}$

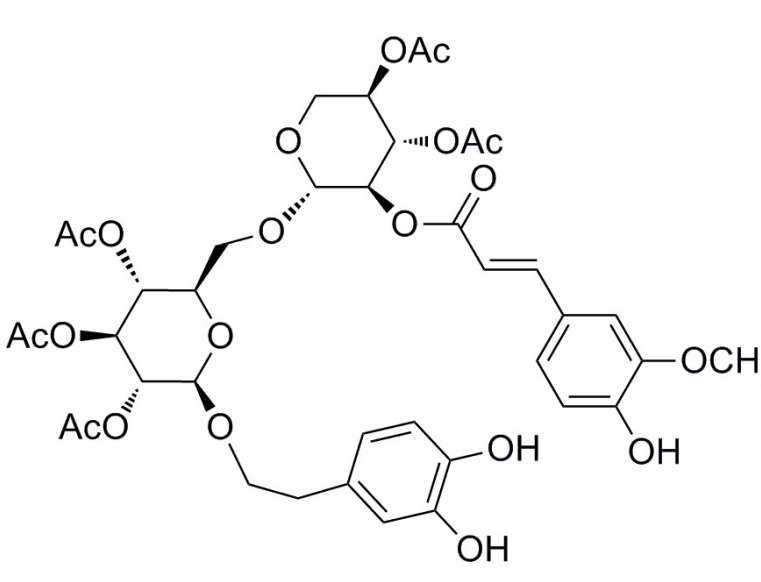

14

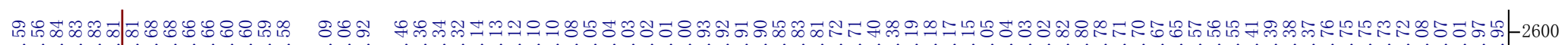

1.

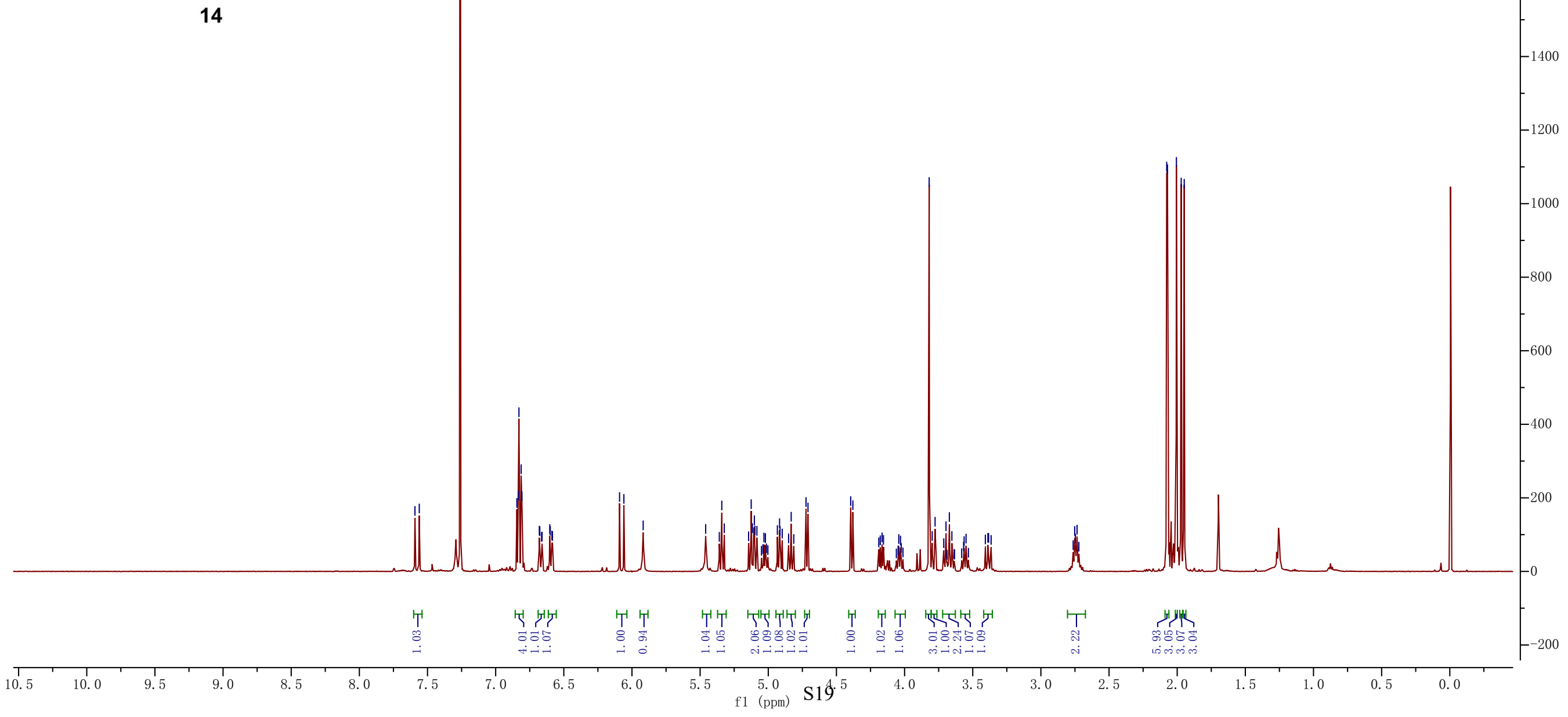




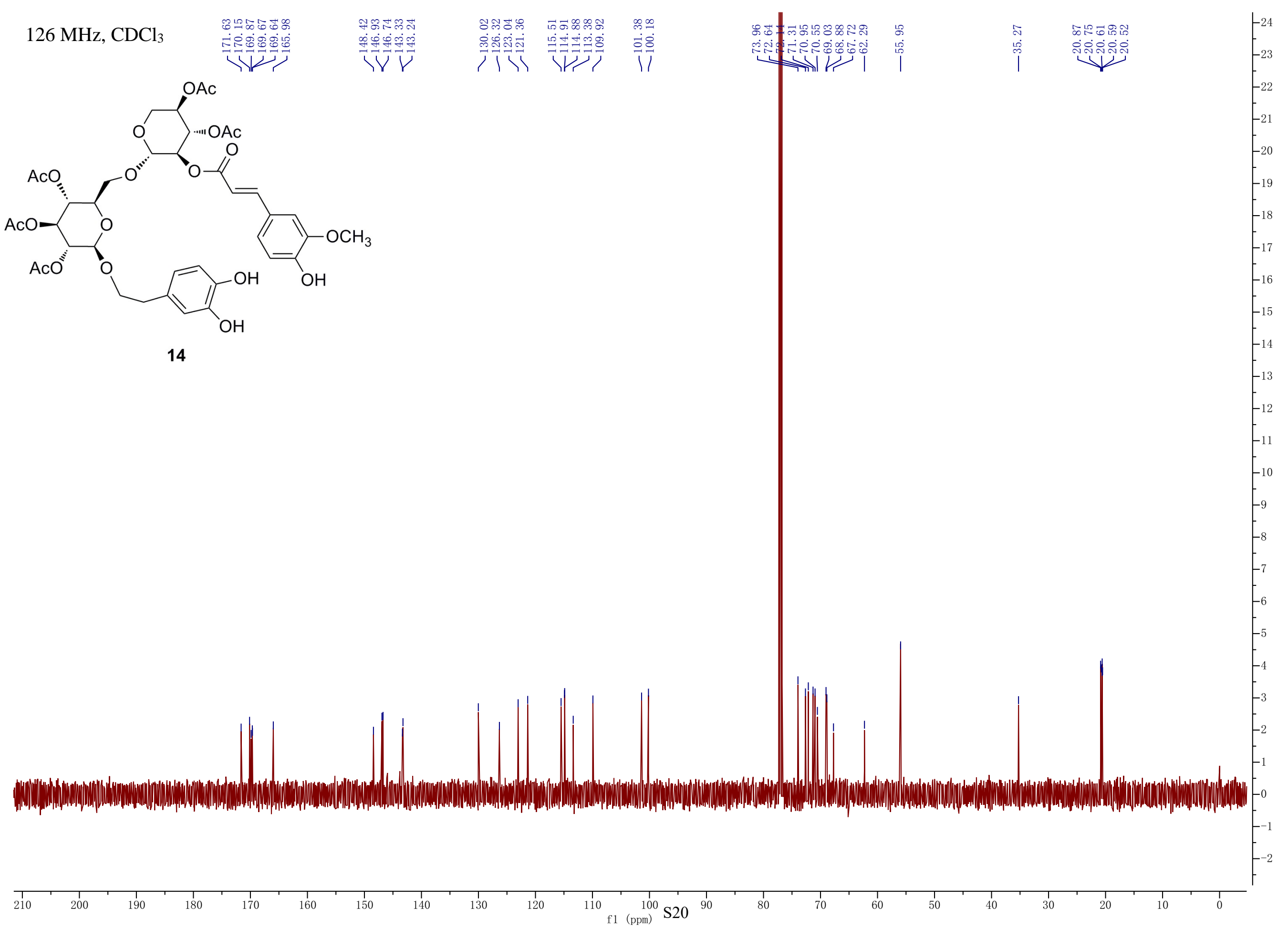




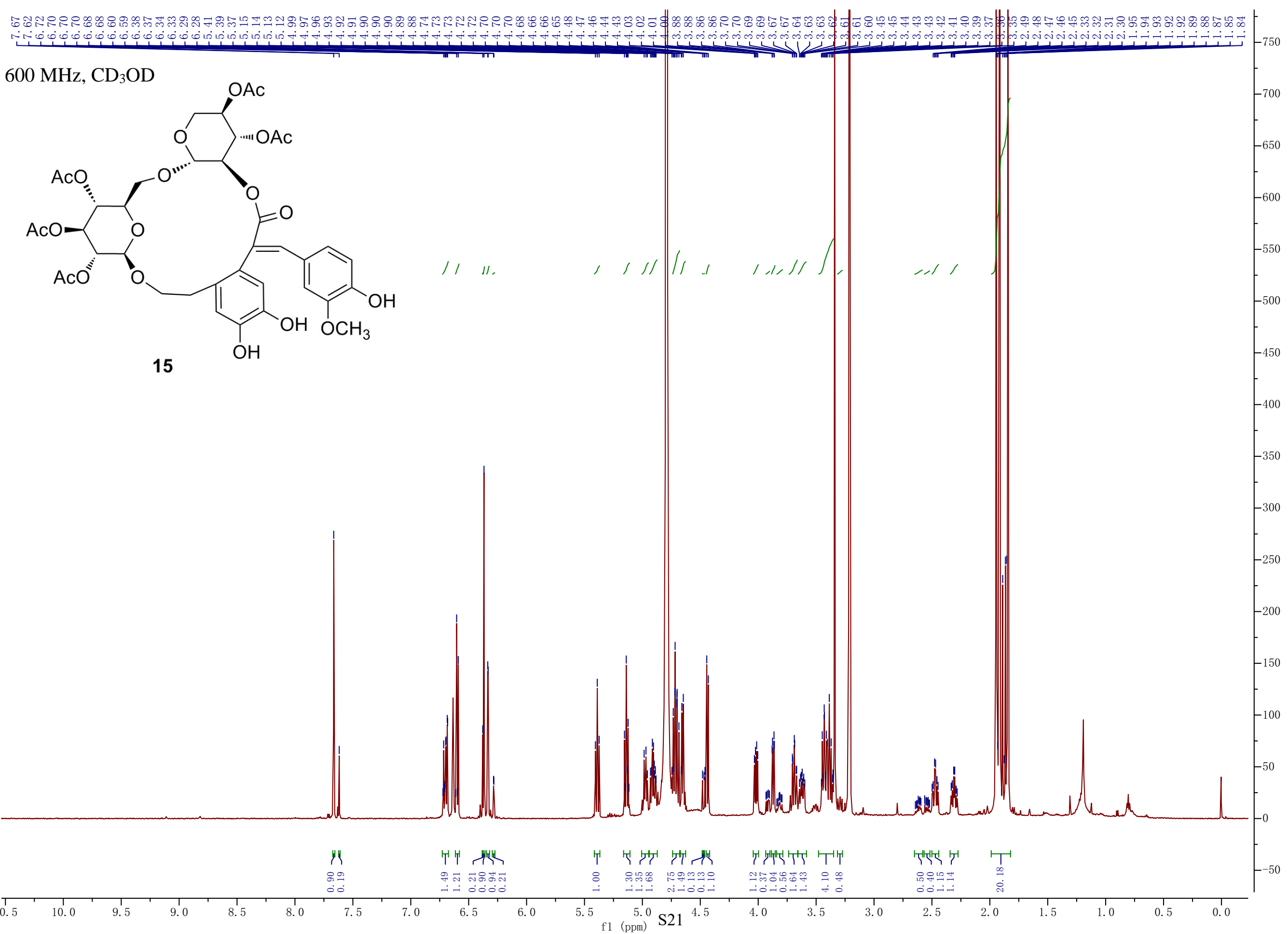




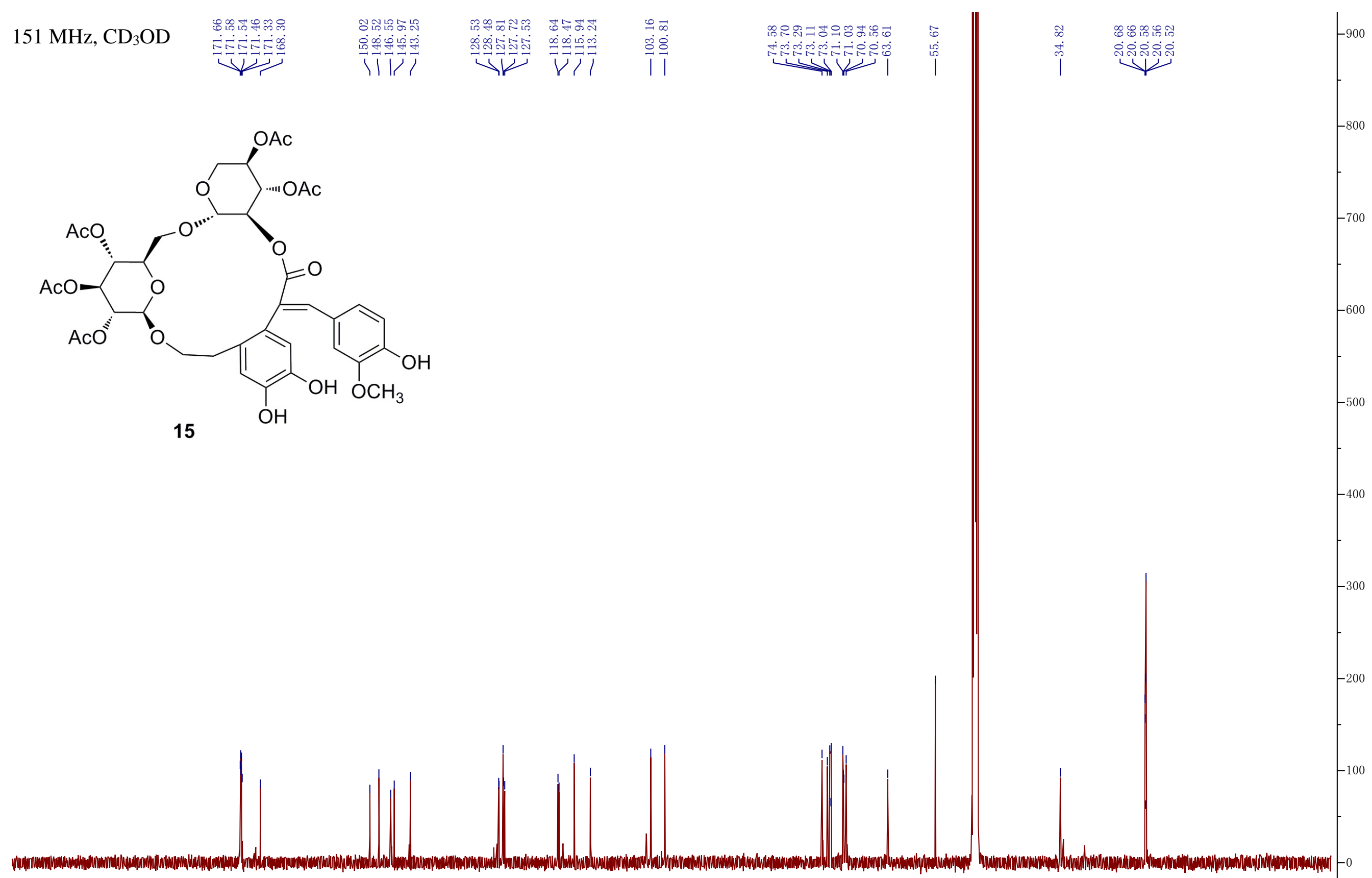




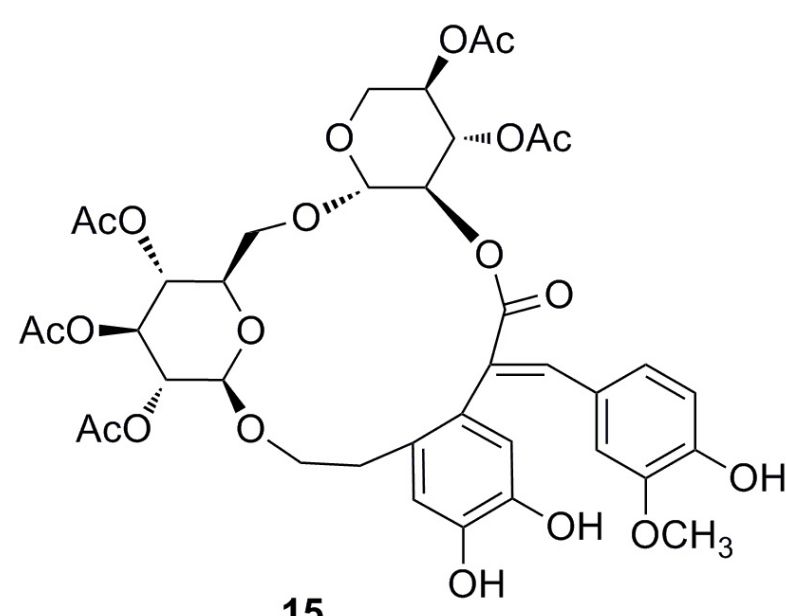

$\int 11$
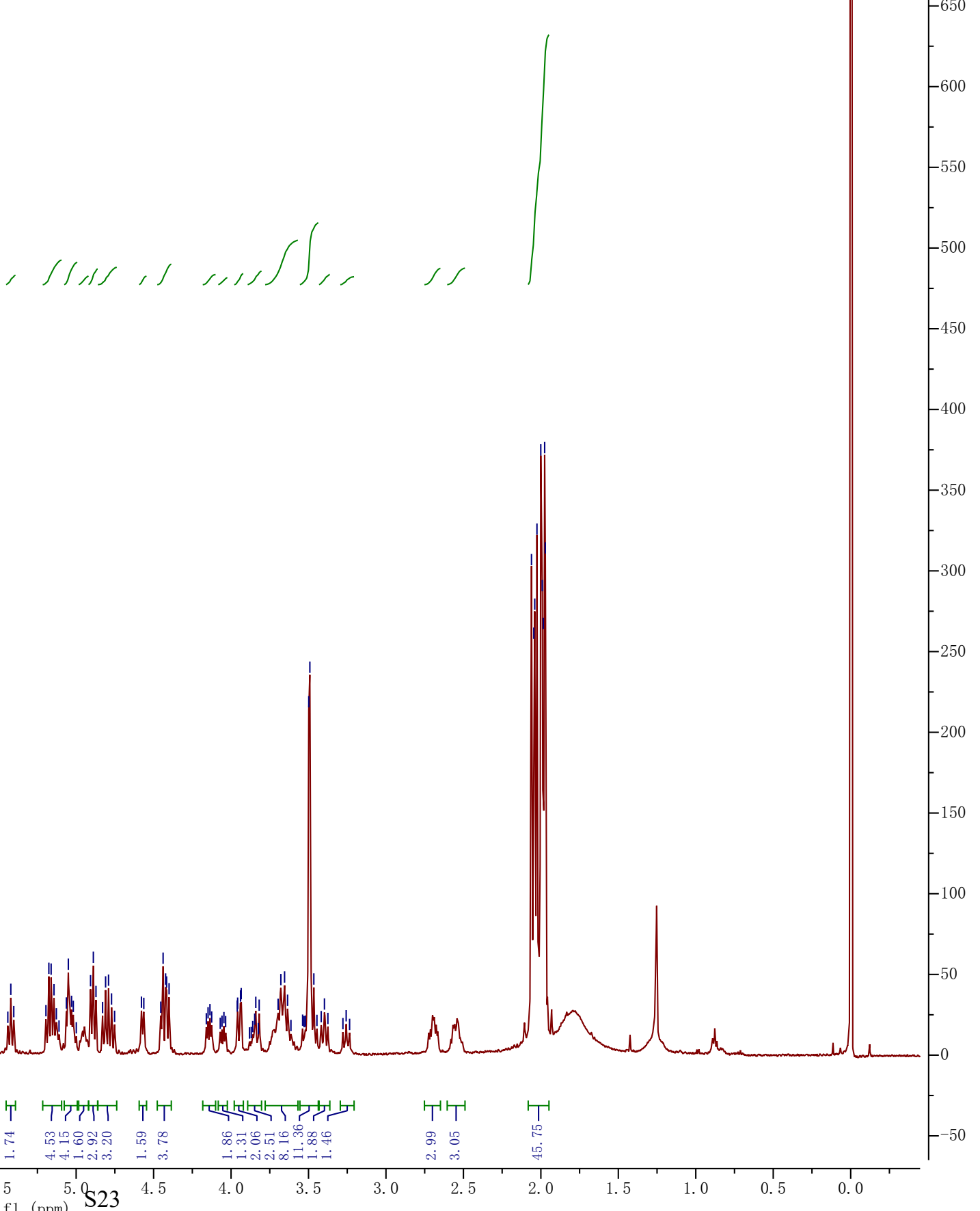


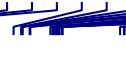

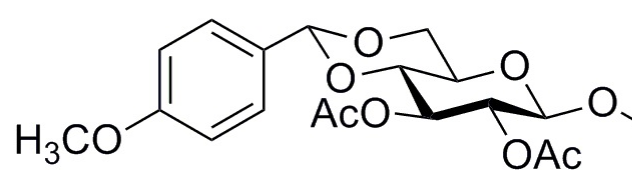

16

OAC

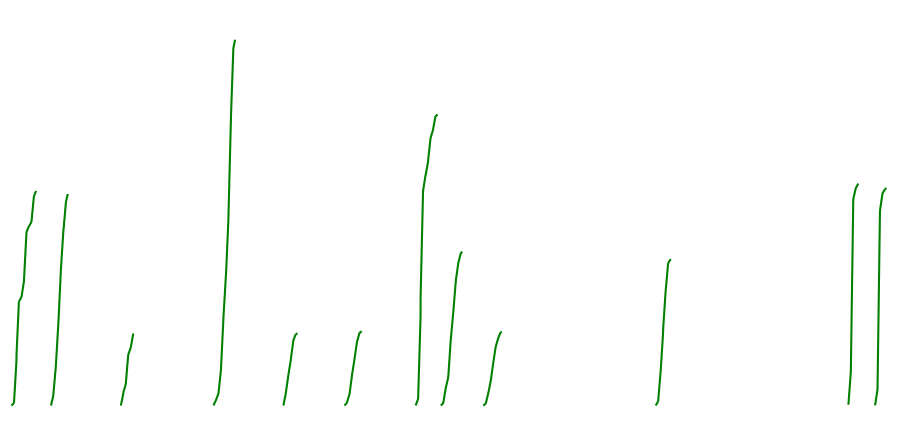

$-1800$

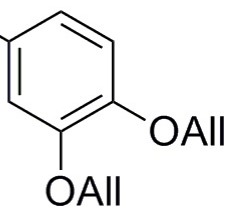

11
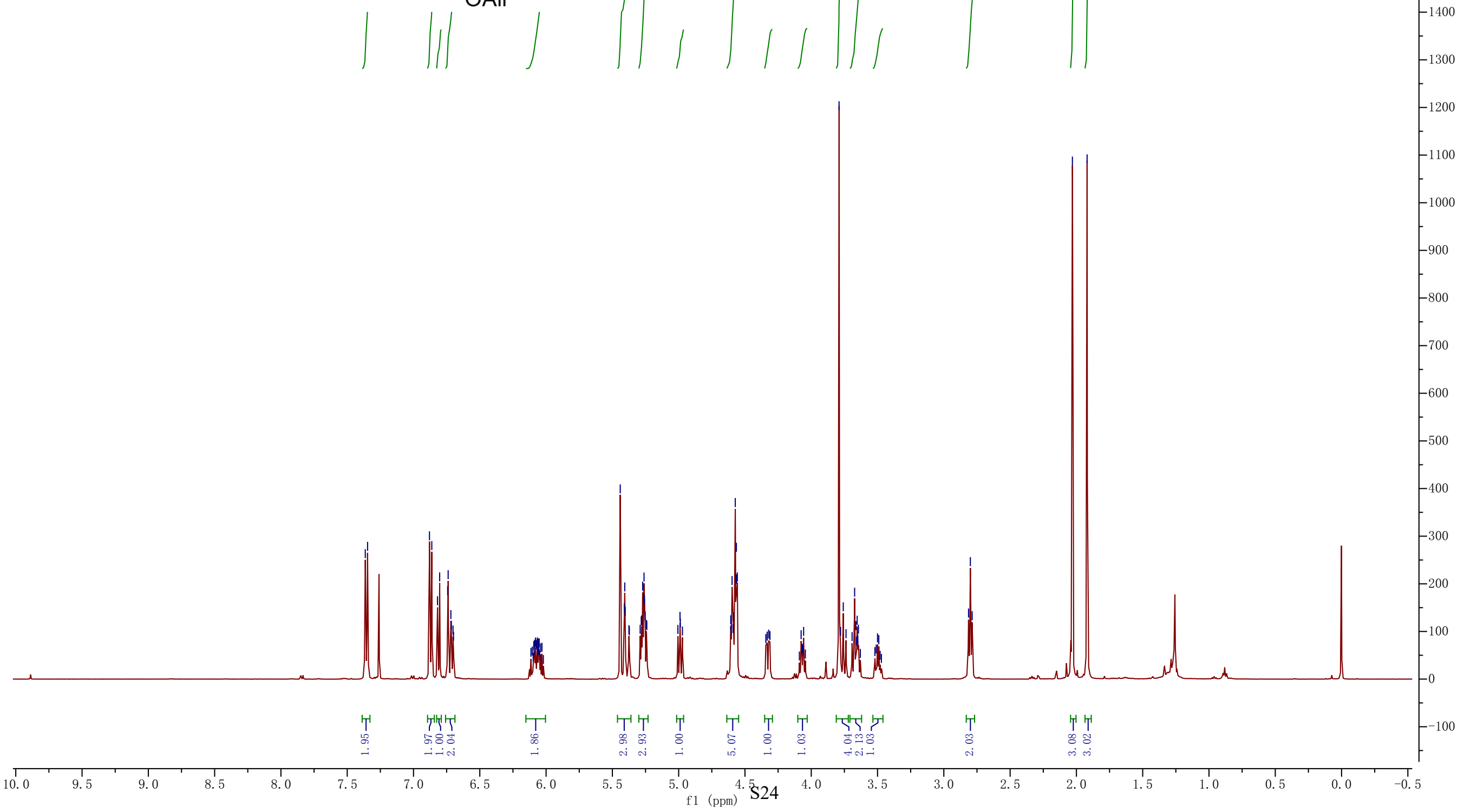


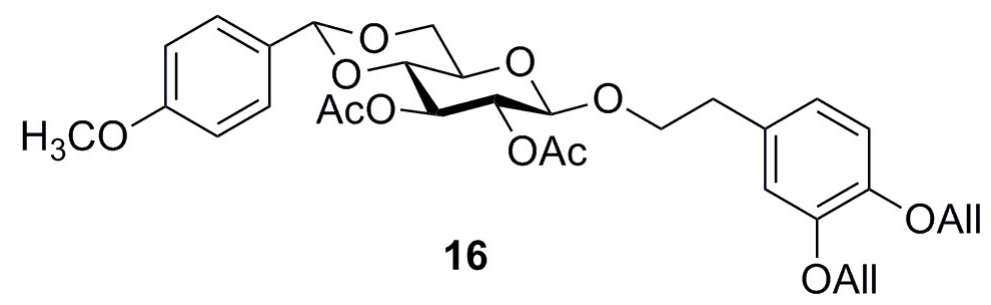



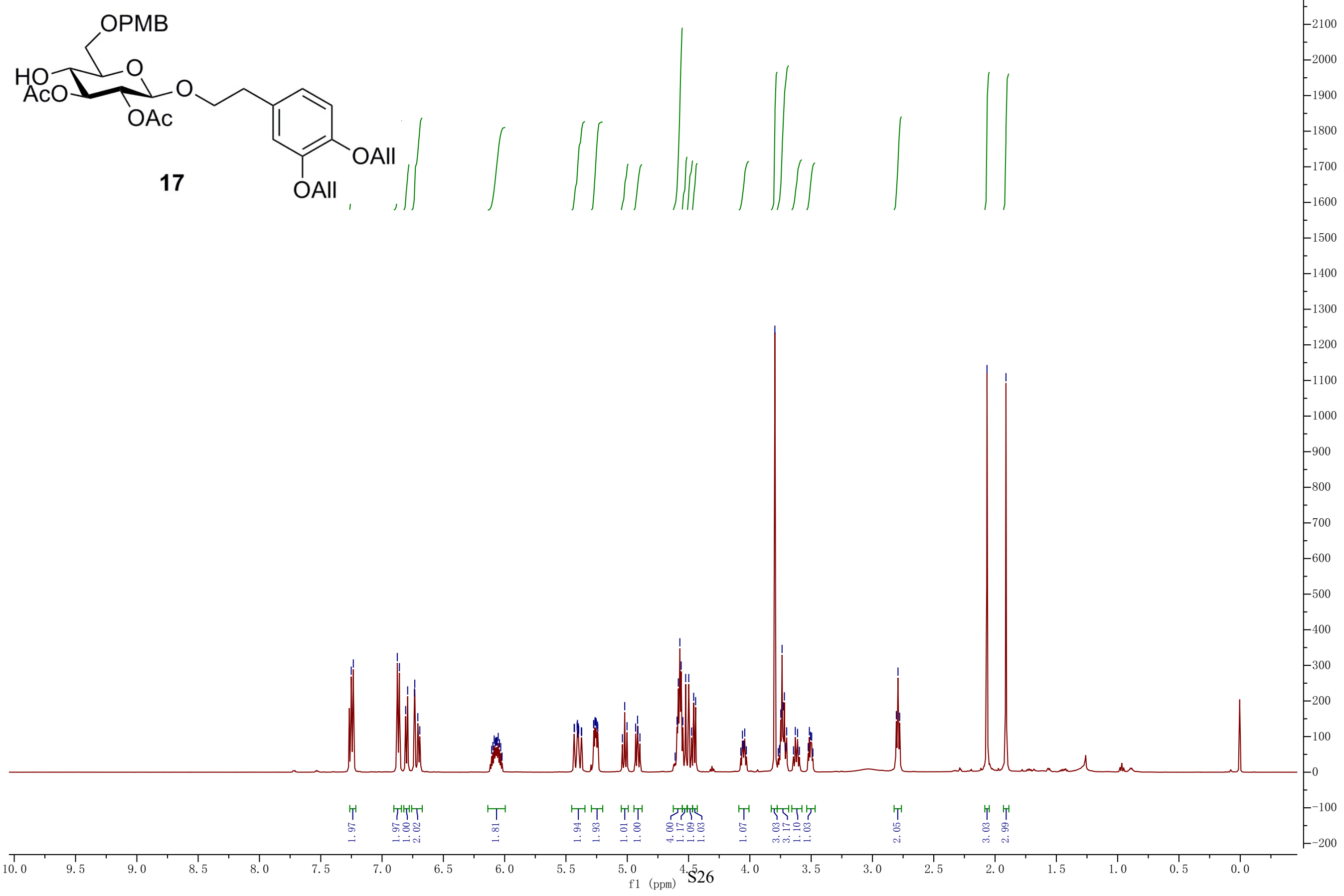


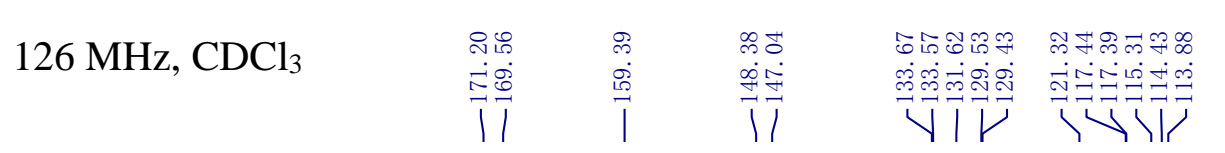

1
0
0
0

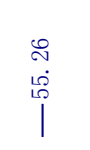

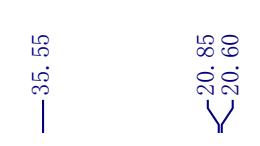

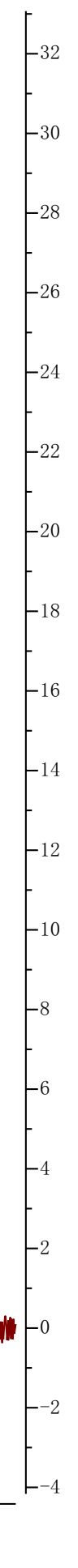



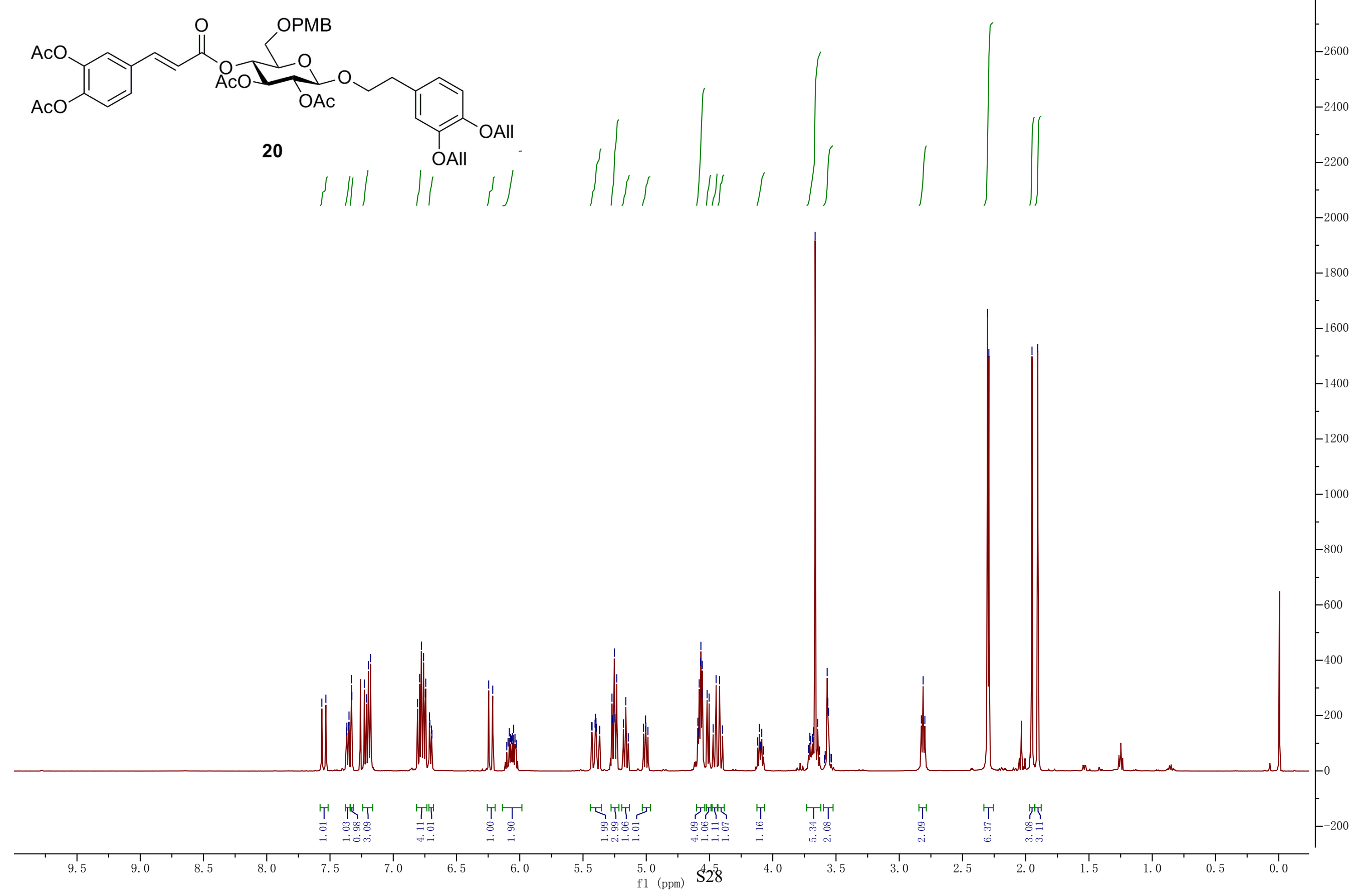


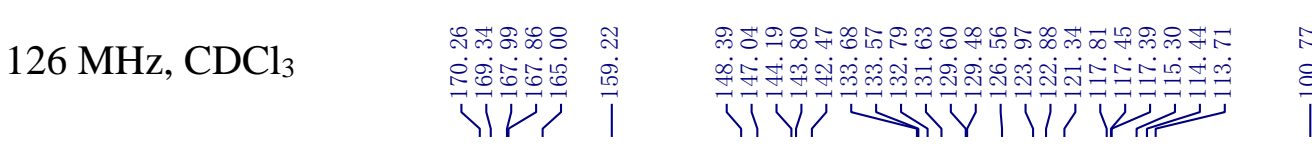

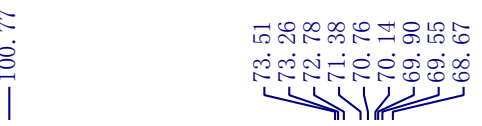

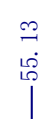

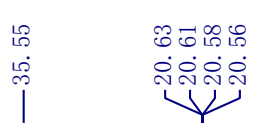
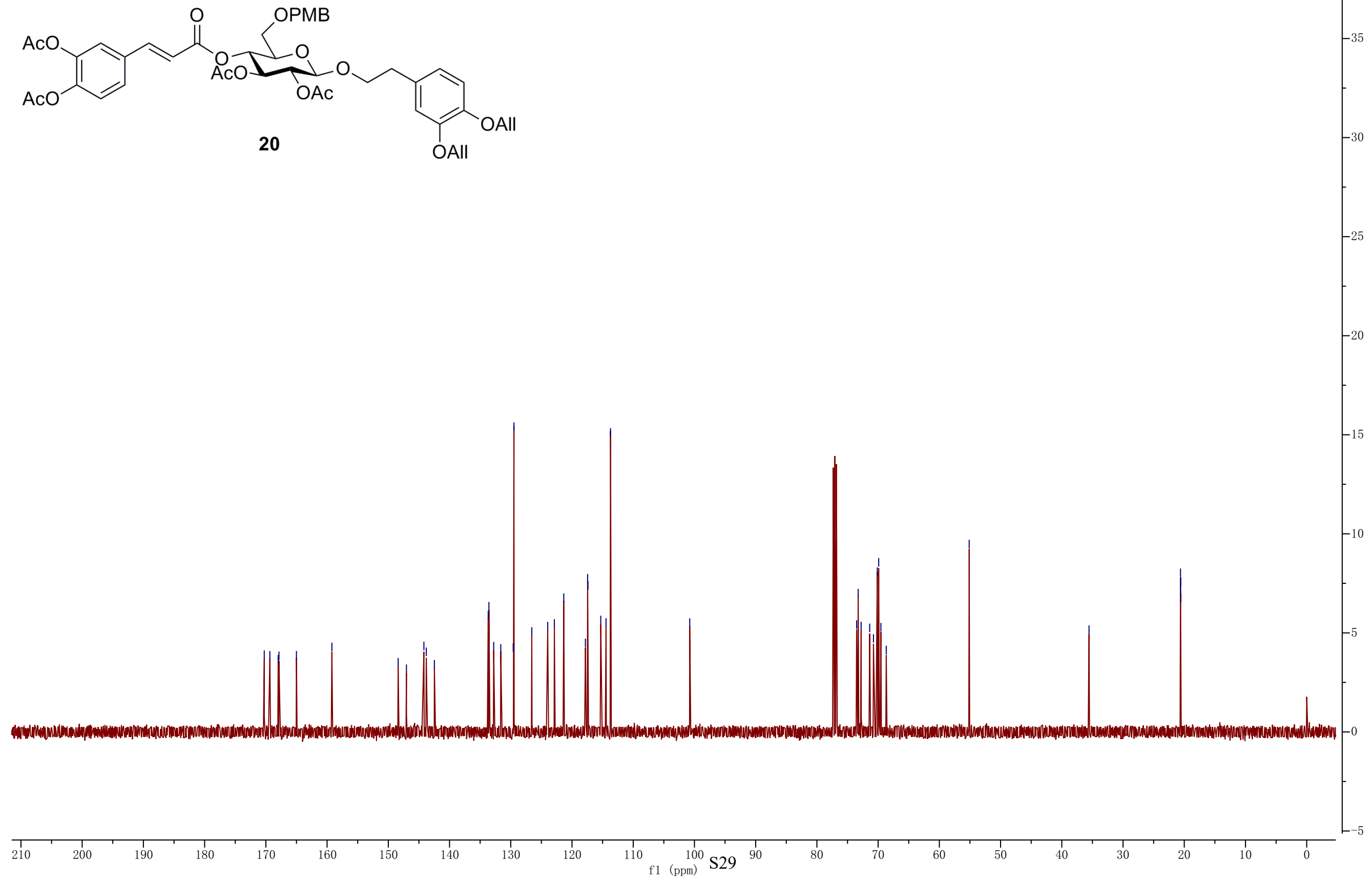


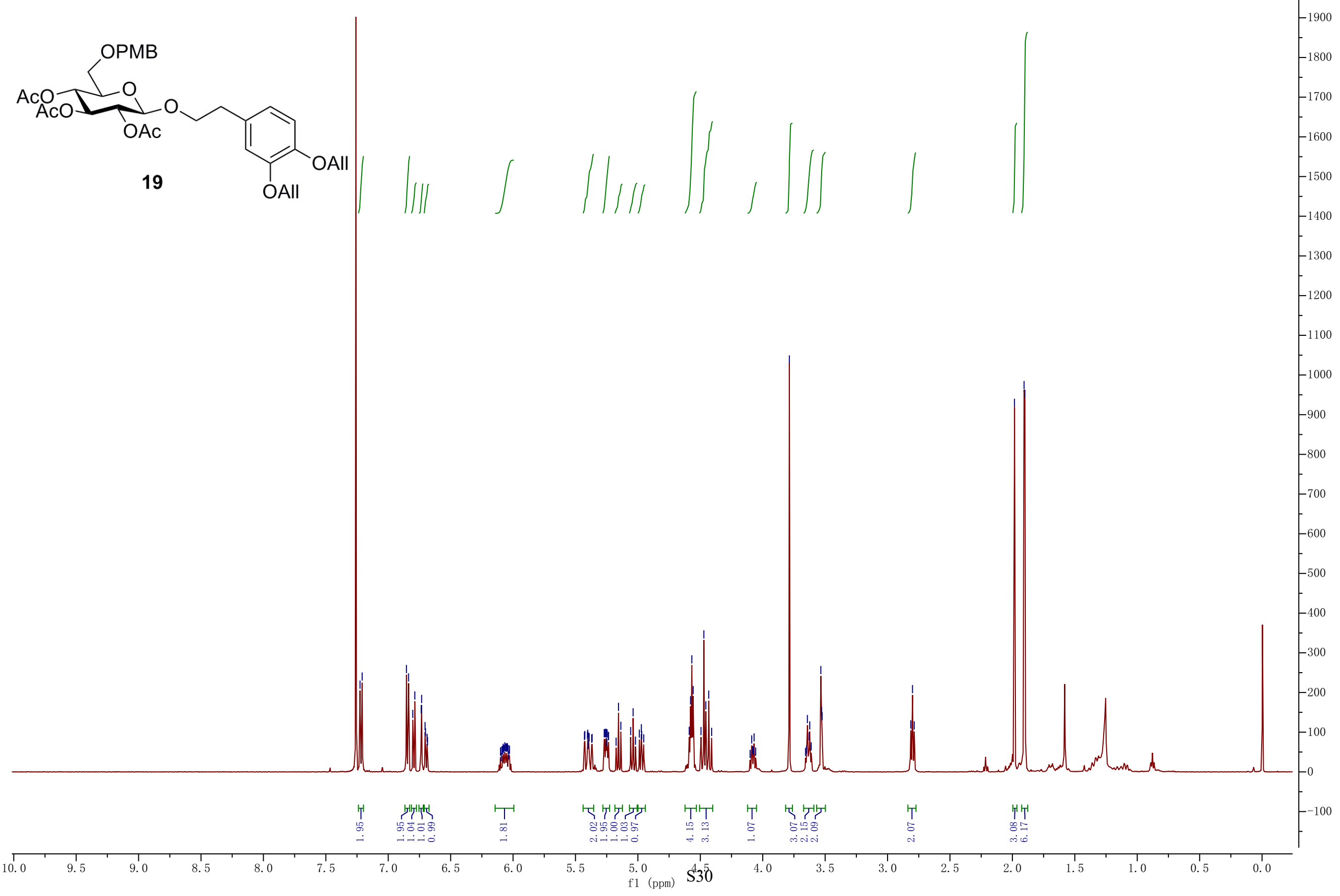


$126 \mathrm{MHz}, \mathrm{CDCl}_{3}$

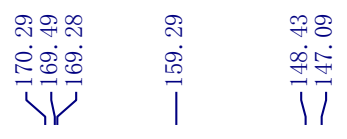

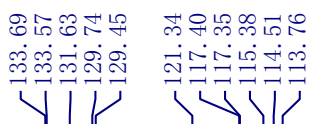

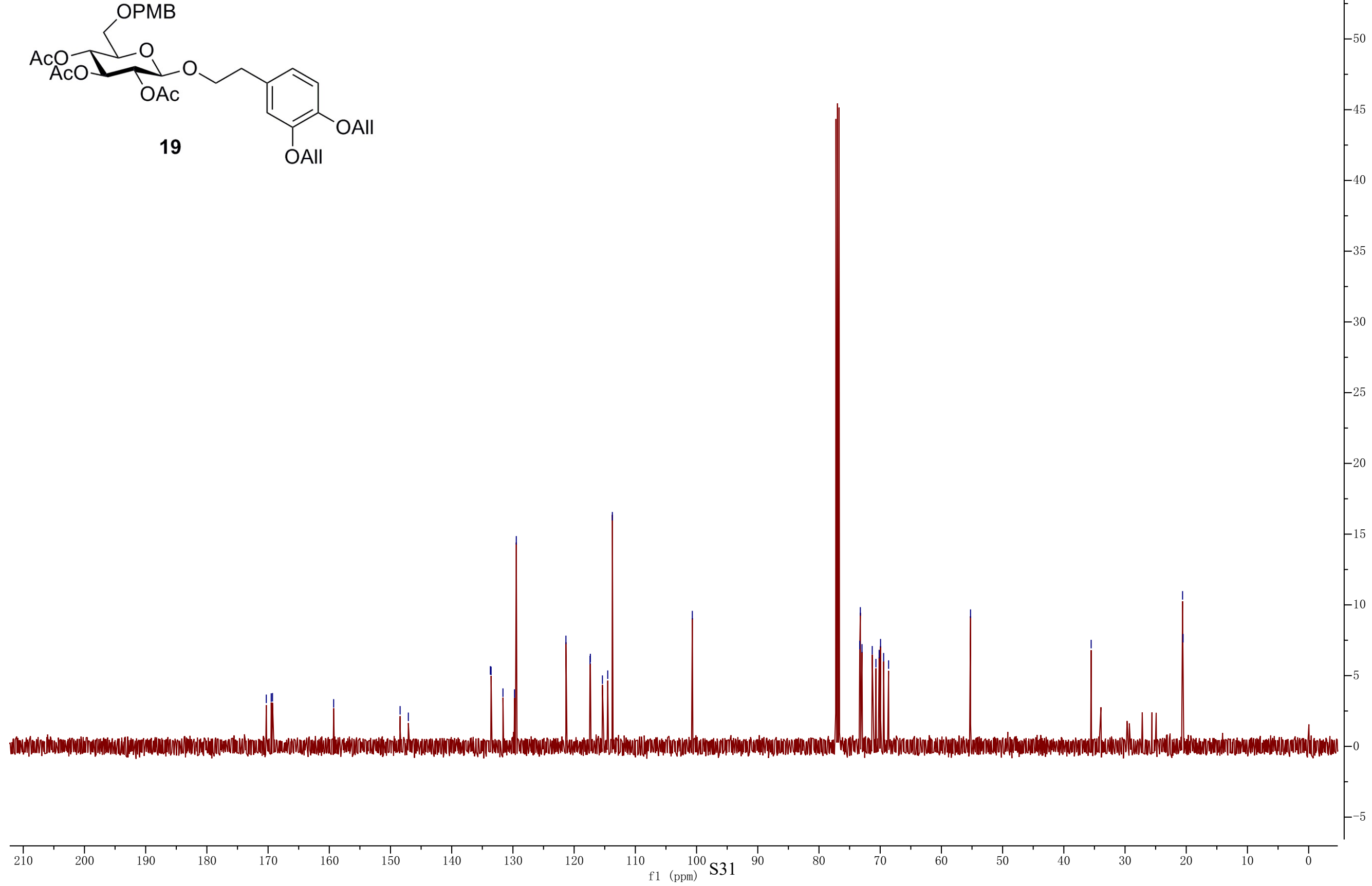

19 


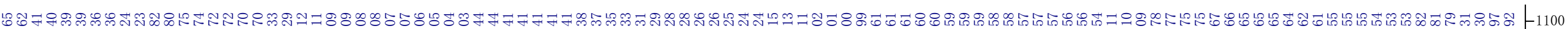

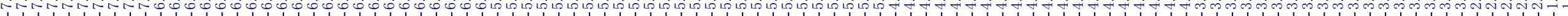
$500 \mathrm{MHz}, \mathrm{CDCl}_{3}$
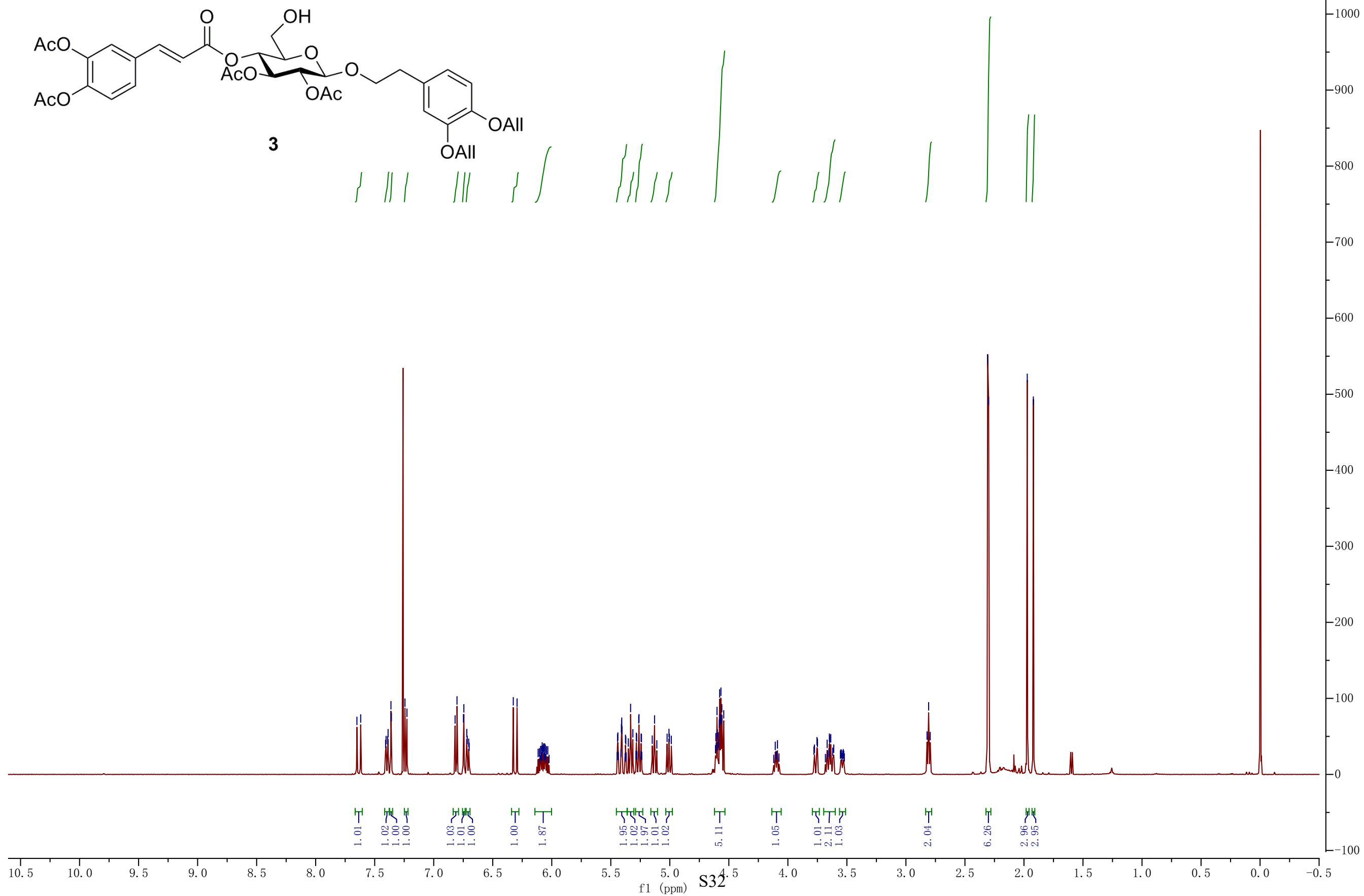

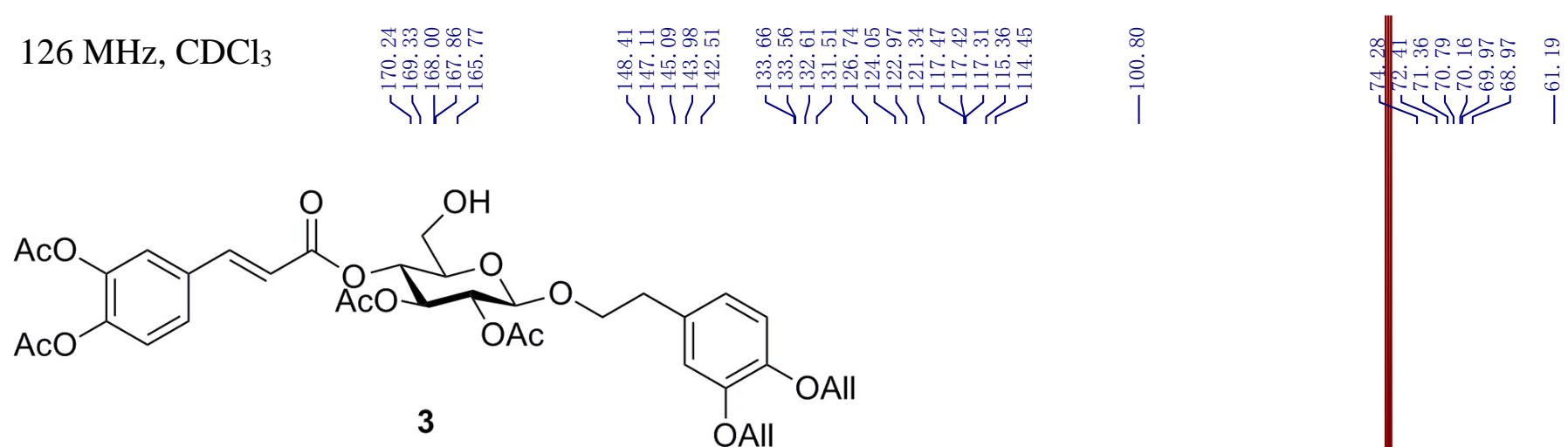

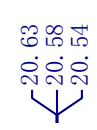

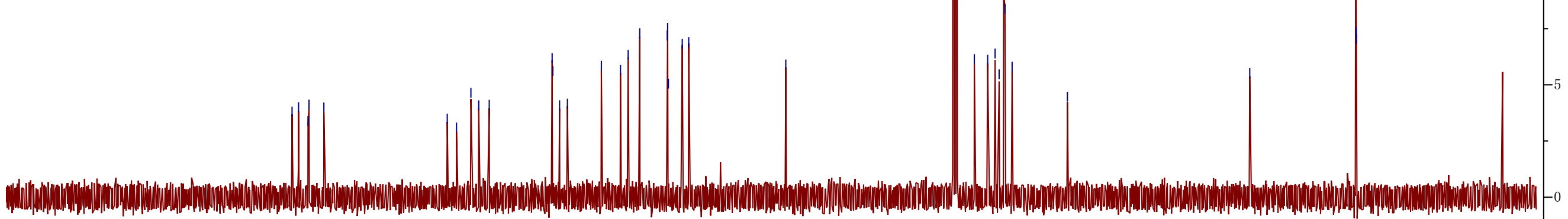



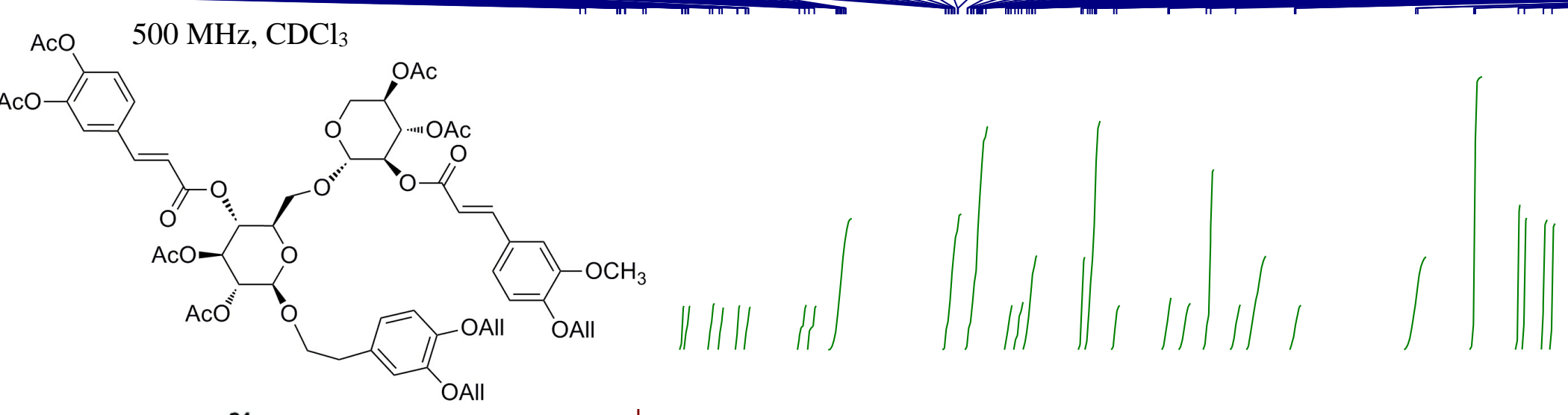

21
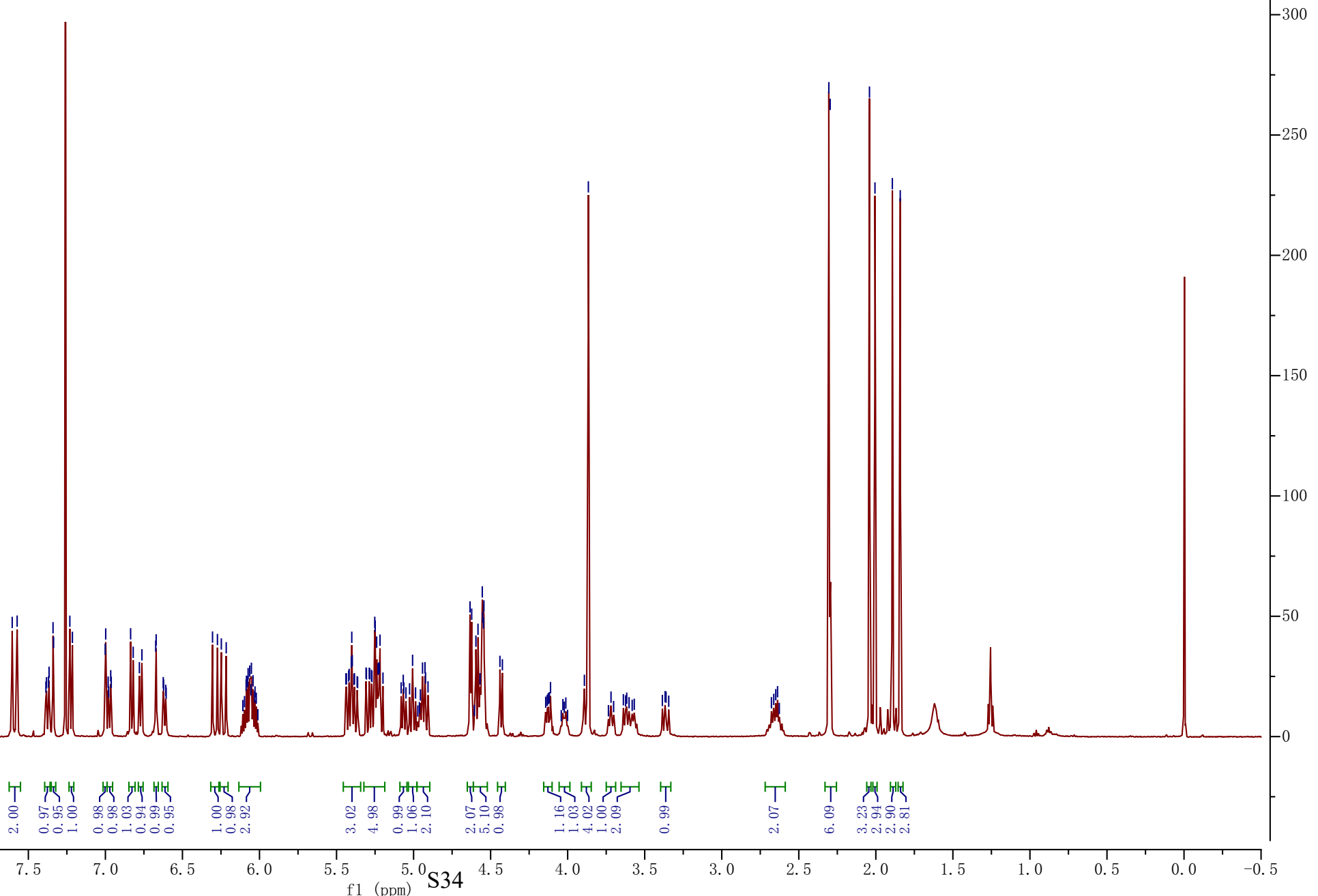
$126 \mathrm{MHz}, \mathrm{CDCl}_{3}$

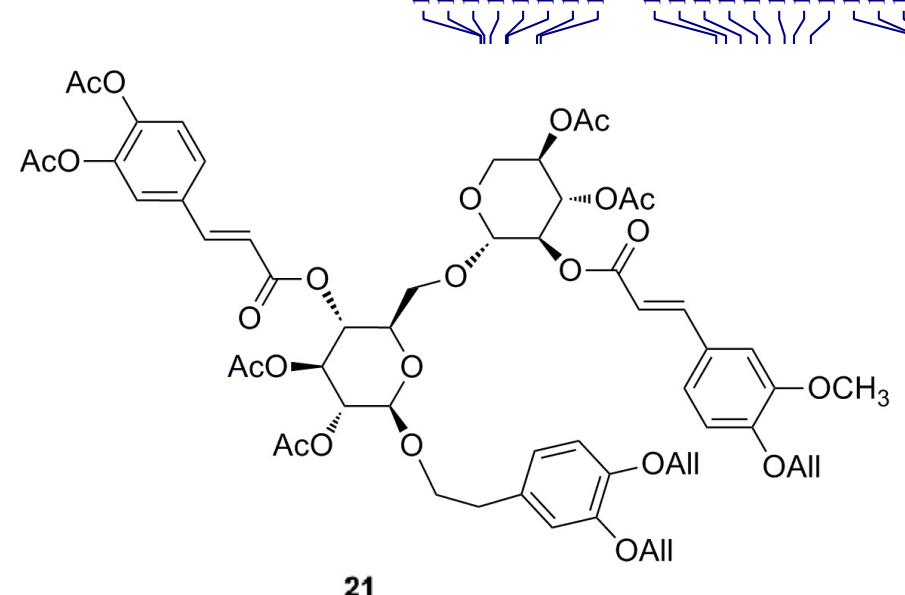

21 
$500 \mathrm{MHz}, \mathrm{CDCl}_{3}$

두요 ن

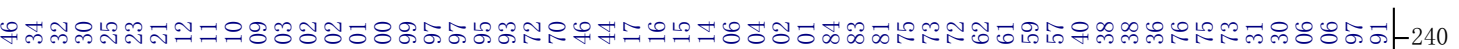
The

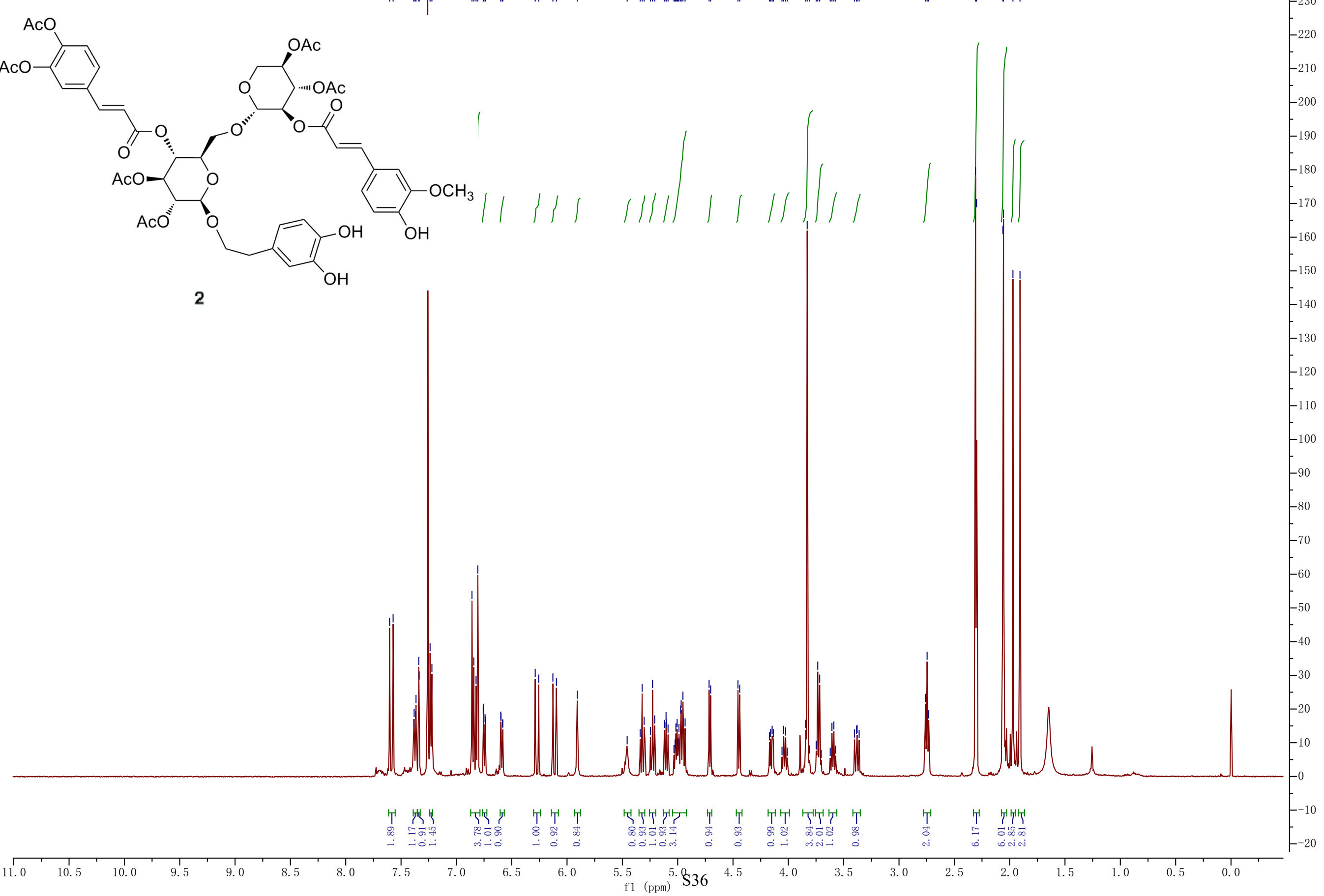




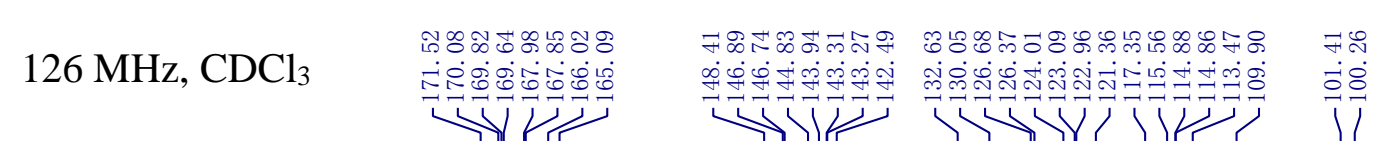
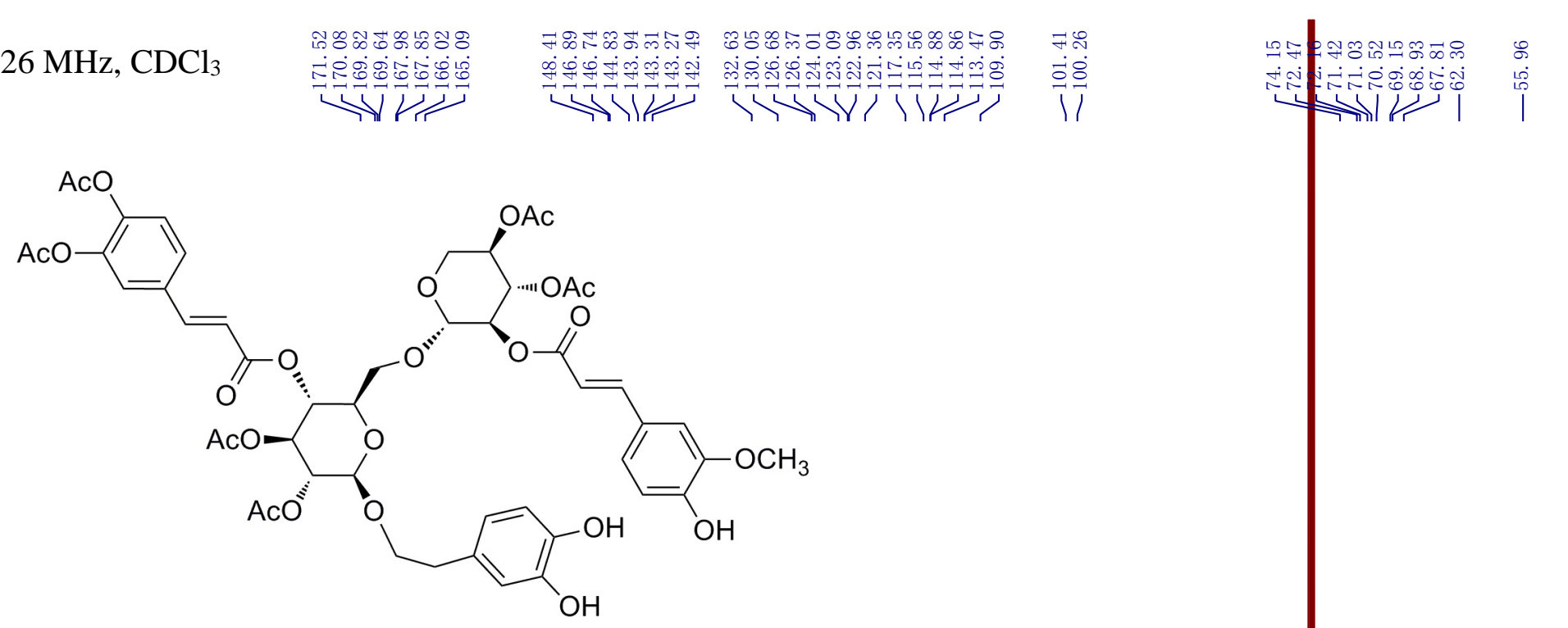

2

$$
\text { . }
$$




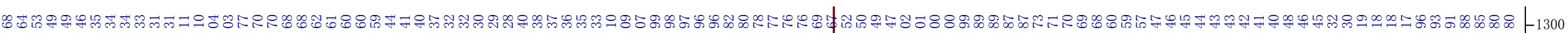

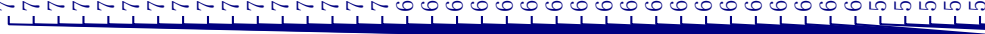
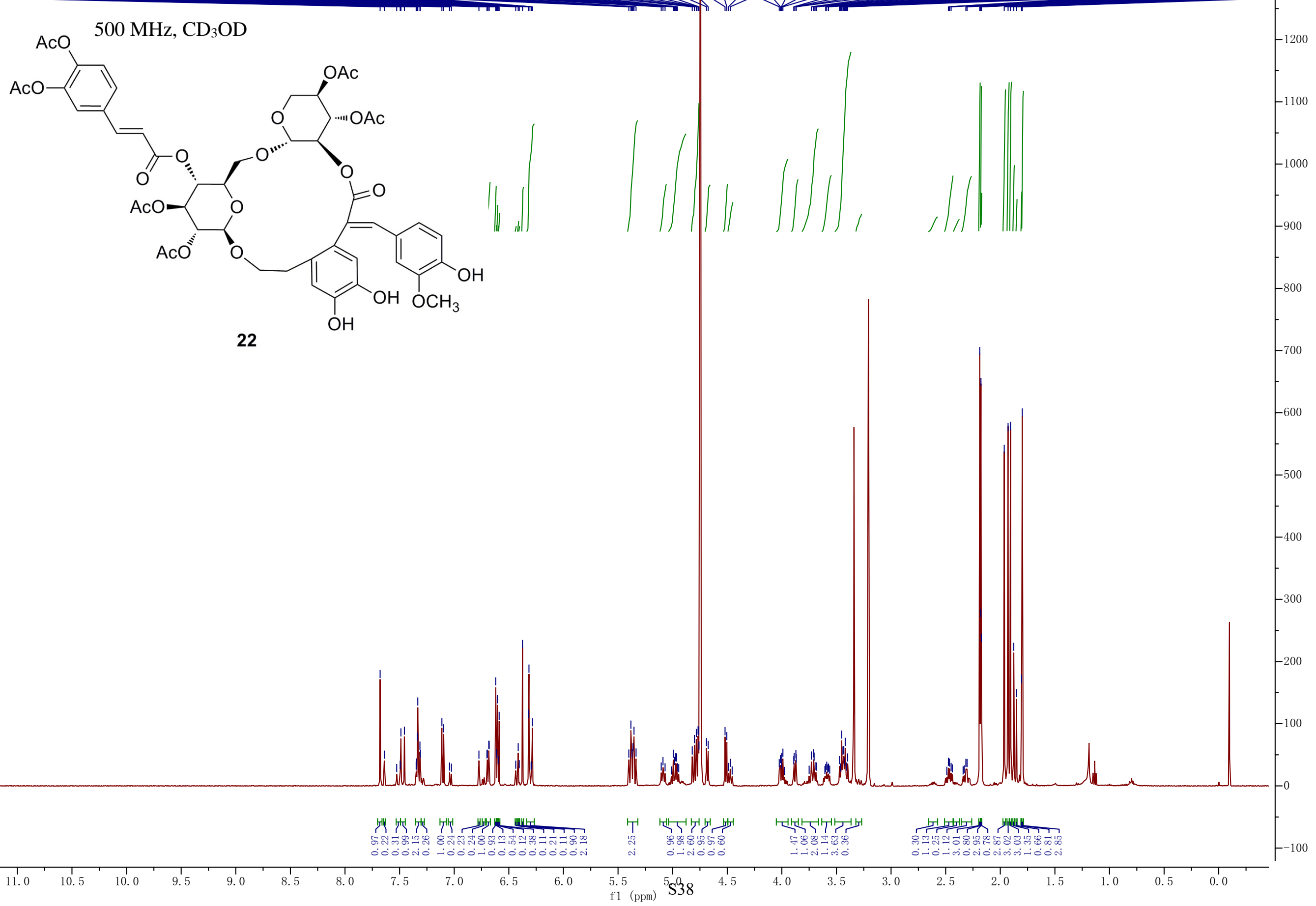
$151 \mathrm{MHz}, \mathrm{CD}_{3} \mathrm{OD}$

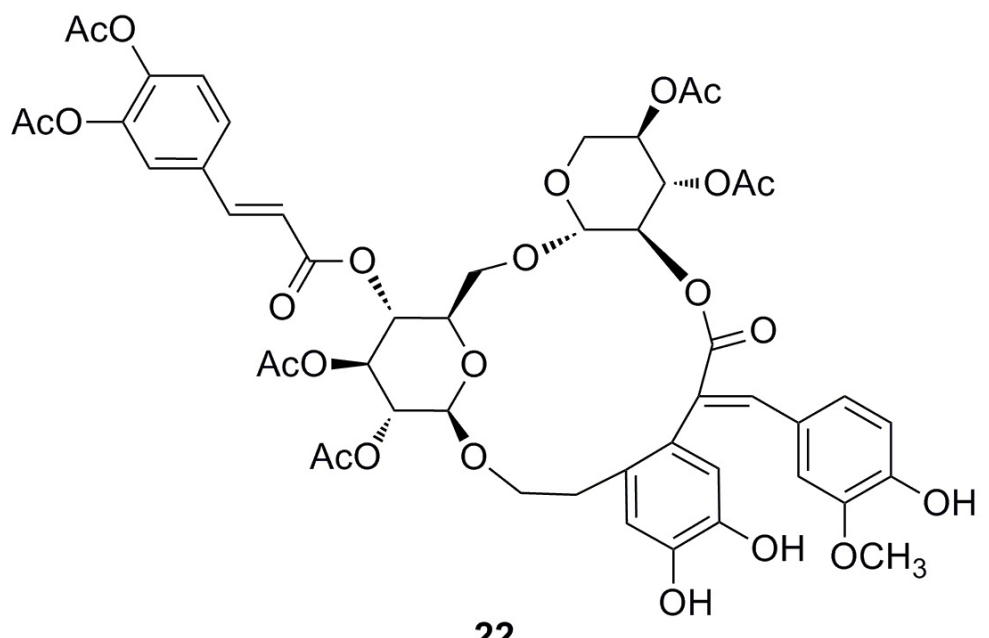

22
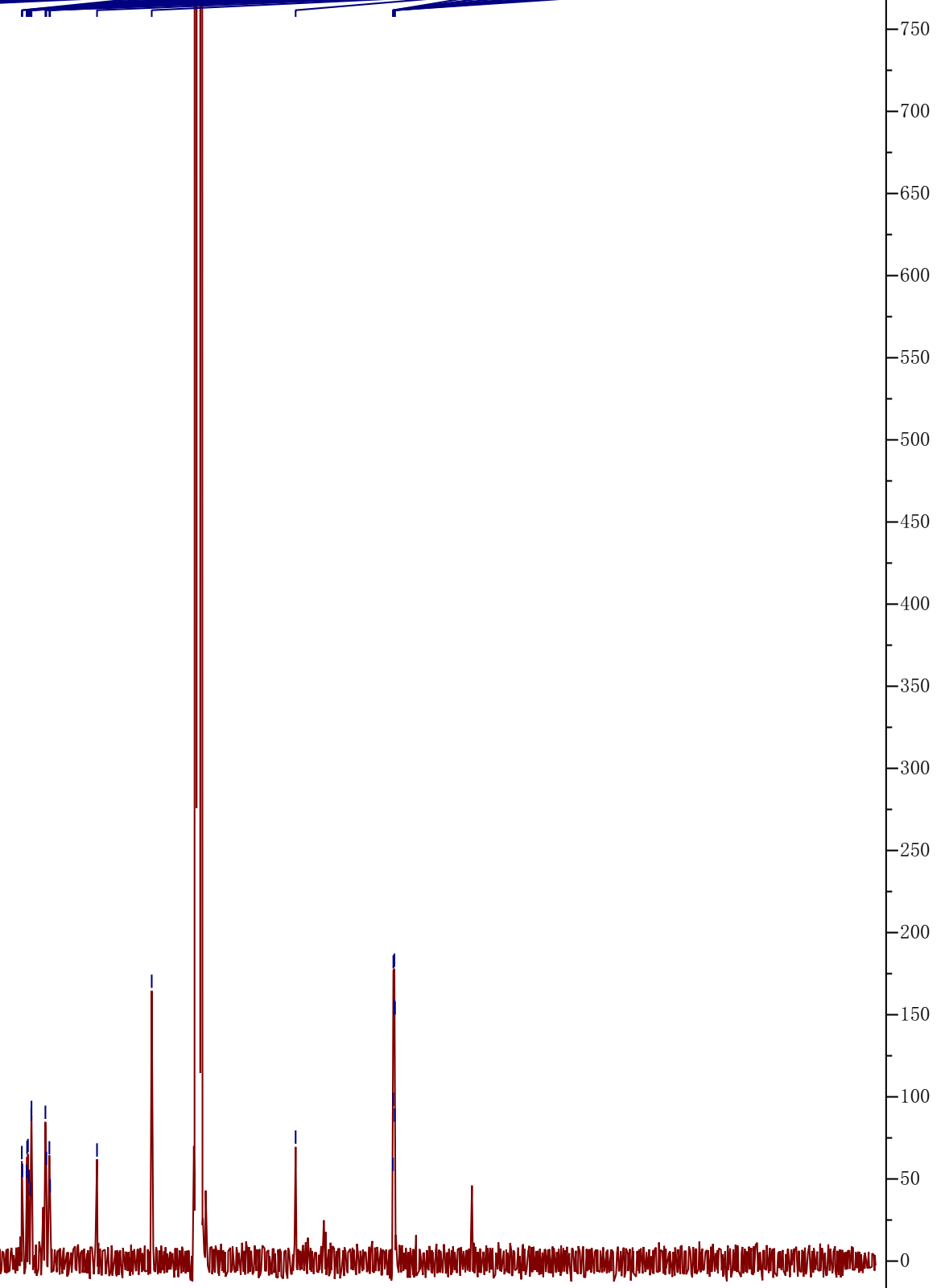

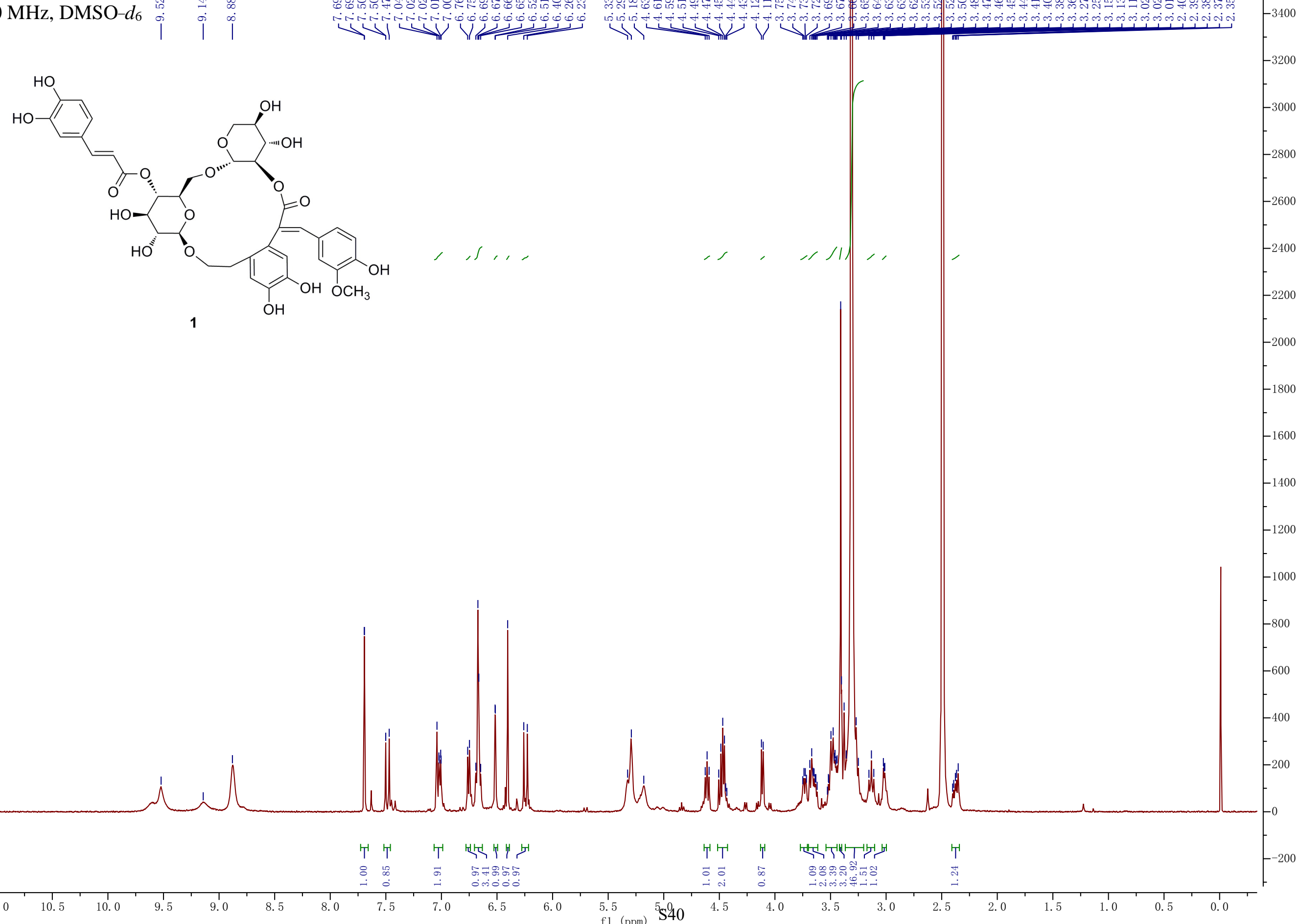

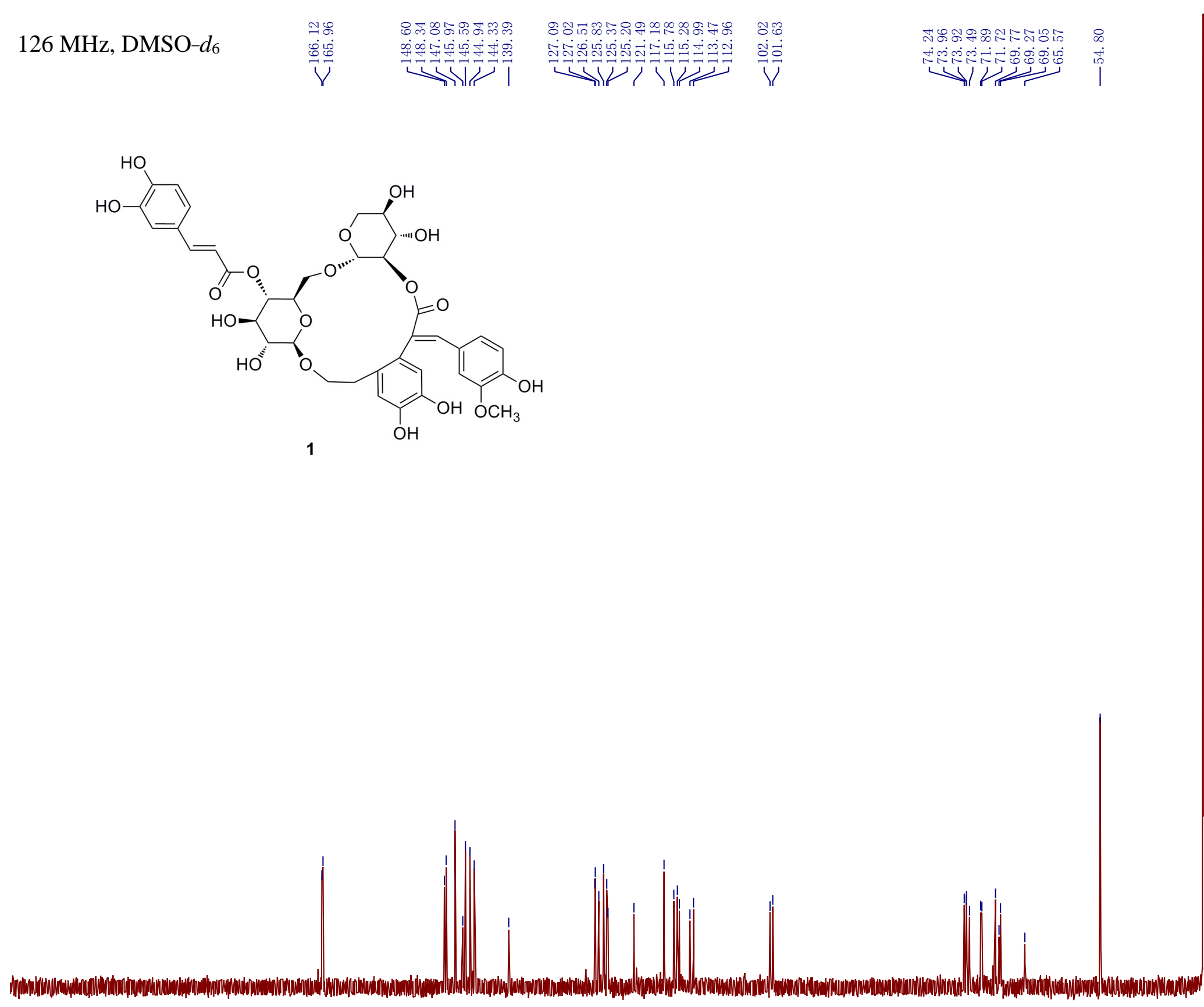
Final report from the MD simulations run on Forsythenethoside A (1), Macrocycle 15_conformer A and Macrocycle 15_conformer B

\section{Forsythenethoside A (1)}

Final potential and kinetic energy $=\quad 803.72 \quad 310.57 \mathrm{~kJ} / \mathrm{mol}$ CPU Time $=995.0 \mathrm{sec}$

Average potential energy $=\quad 813.47 \mathrm{~kJ} / \mathrm{mol}$

Average kinetic energy $=299.76 \mathrm{~kJ} / \mathrm{mol}($ Av temperature $=298.0 \mathrm{deg} \mathrm{K})$

Average total energy $=1113.23 \mathrm{~kJ} / \mathrm{mol}(\operatorname{Std~dev}=10.78 \mathrm{~kJ} / \mathrm{mol})$

Average potential energy $\langle\mathrm{H}>$ scaled to $298.0 \mathrm{deg} \mathrm{K}=$
Av stretch
$102.84 \mathrm{~kJ} / \mathrm{mol}$
Av bend
$179.42 \mathrm{~kJ} / \mathrm{mol}$
Av torsion
$94.66 \mathrm{~kJ} / \mathrm{mol}$
Av van der Waals $201.73 \mathrm{~kJ} / \mathrm{mol}$
Av electrostatic $\quad 233.17 \mathrm{~kJ} / \mathrm{mol}$
Av hydrogen bond $\quad-1.91 \mathrm{~kJ} / \mathrm{mol}$
Av cross terms $\quad 1.00 \mathrm{~kJ} / \mathrm{mol}$
Av solvation $1 \quad 0.00 \mathrm{~kJ} / \mathrm{mol}$
Av solvation $2 \quad 0.00 \mathrm{~kJ} / \mathrm{mol}$

$813.51 \mathrm{~kJ} / \mathrm{mol}$

Macrocycle 15_conformer A

Final potential and kinetic energy $=435.24 \quad 294.38 \mathrm{~kJ} / \mathrm{mol}$

Average potential energy $=\quad 439.84 \mathrm{~kJ} / \mathrm{mol}$

Average kinetic energy $=320.70 \mathrm{~kJ} / \mathrm{mol}($ Av temperature $=297.8 \mathrm{deg} \mathrm{K})$

Average total energy $=760.54 \mathrm{~kJ} / \mathrm{mol}($ Std dev $=15.58 \mathrm{~kJ} / \mathrm{mol})$

Average potential energy $\langle\mathrm{H}>$ scaled to $298.0 \mathrm{deg} \mathrm{K}=\quad 440.00 \mathrm{~kJ} / \mathrm{mol}$
Av stretch $\quad 99.50 \mathrm{~kJ} / \mathrm{mol}$
Av bend $\quad 237.88 \mathrm{~kJ} / \mathrm{mol}$
Av torsion $\quad 87.46 \mathrm{~kJ} / \mathrm{mol}$
Av van der Waals $186.30 \mathrm{~kJ} / \mathrm{mol}$
Av electrostatic $\quad-83.09 \mathrm{~kJ} / \mathrm{mol}$
Av hydrogen bond $\quad-1.16 \mathrm{~kJ} / \mathrm{mol}$
Av cross terms $\quad 0.42 \mathrm{~kJ} / \mathrm{mol}$
Av solvation $1 \quad-77.68 \mathrm{~kJ} / \mathrm{mol}$
Av solvation $2 \quad-11.27 \mathrm{~kJ} / \mathrm{mol}$

\section{Macrocycle 15_conformer B}

Final potential and kinetic energy $=\quad 539.05 \quad 301.68 \mathrm{~kJ} / \mathrm{mol}$

Average potential energy $=\quad 548.95 \mathrm{~kJ} / \mathrm{mol}$

Average kinetic energy $=320.52 \mathrm{~kJ} / \mathrm{mol}($ Av temperature $=297.7 \mathrm{deg} \mathrm{K})$

Average total energy $=869.46 \mathrm{~kJ} / \mathrm{mol}(\operatorname{Std~dev}=8.93 \mathrm{~kJ} / \mathrm{mol})$

Average potential energy $\langle\mathrm{H}\rangle$ scaled to $298.0 \mathrm{deg} \mathrm{K}=549.29 \mathrm{~kJ} / \mathrm{mol}$

Av stretch $\quad 105.23 \mathrm{~kJ} / \mathrm{mol}$

Av bend $\quad 253.58 \mathrm{~kJ} / \mathrm{mol}$

Av torsion $\quad 138.14 \mathrm{~kJ} / \mathrm{mol}$

Av van der Waals $188.79 \mathrm{~kJ} / \mathrm{mol}$

Av electrostatic $\quad-69.62 \mathrm{~kJ} / \mathrm{mol}$

Av hydrogen bond $\quad-0.90 \mathrm{~kJ} / \mathrm{mol}$

Av cross terms $\quad 0.28 \mathrm{~kJ} / \mathrm{mol}$

Av solvation $1 \quad-71.69 \mathrm{~kJ} / \mathrm{mol}$

Av solvation $2 \quad-11.20 \mathrm{~kJ} / \mathrm{mol}$ 
Data for Forsythenethoside A (1)

96

campione

\begin{tabular}{|c|c|c|c|}
\hline $\mathrm{C}$ & -3.37300 & -1.03830 & -10.44950 \\
\hline $\mathrm{C}$ & -2.52110 & -0.13370 & -9.53240 \\
\hline $\mathrm{C}$ & -2.46960 & 1.29430 & -10.10710 \\
\hline $\mathrm{C}$ & -2.00530 & 1.27230 & -11.57090 \\
\hline $\mathrm{C}$ & -2.92870 & 0.32080 & -12.35900 \\
\hline $\mathrm{O}$ & -2.86300 & -0.95700 & -11.74320 \\
\hline $\mathrm{O}$ & -1.55320 & 2.02340 & -9.30190 \\
\hline $\mathrm{O}$ & -2.14690 & 2.59600 & -12.07530 \\
\hline C & -2.51020 & 0.09510 & -13.81490 \\
\hline $\mathrm{H}$ & -1.43140 & 2.87460 & -9.69720 \\
\hline $\mathrm{H}$ & -1.52090 & -0.56360 & -9.45210 \\
\hline $\mathrm{H}$ & -3.45830 & 1.75560 & -10.04590 \\
\hline $\mathrm{H}$ & -0.97720 & 0.90830 & -11.61740 \\
\hline $\mathrm{H}$ & -3.95710 & 0.68620 & -12.33730 \\
\hline $\mathrm{H}$ & -2.55530 & 1.02640 & -14.37990 \\
\hline $\mathrm{H}$ & -1.48590 & -0.27780 & -13.86270 \\
\hline $\mathrm{O}$ & -3.20570 & -2.34160 & -10.00920 \\
\hline $\mathrm{H}$ & -4.42860 & -0.75810 & -10.42660 \\
\hline $\mathrm{C}$ & -3.14550 & -1.23370 & -15.65800 \\
\hline $\mathrm{C}$ & -4.01980 & -2.48000 & -15.95640 \\
\hline $\mathrm{C}$ & -3.86840 & -2.85280 & -17.44290 \\
\hline $\mathrm{C}$ & -4.16330 & -1.63280 & -18.31740 \\
\hline $\mathrm{C}$ & -3.25610 & -0.46870 & -17.90590 \\
\hline $\mathrm{O}$ & -3.47650 & -0.19020 & -16.52750 \\
\hline $\mathrm{O}$ & -4.80980 & -3.84800 & -17.82170 \\
\hline $\mathrm{O}$ & -3.91580 & -2.02640 & -19.64550 \\
\hline $\mathrm{H}$ & -4.86470 & -4.48050 & -17.11880 \\
\hline $\mathrm{H}$ & -5.05660 & -2.23910 & -15.71620 \\
\hline $\mathrm{H}$ & -2.85500 & -3.21370 & -17.63200 \\
\hline $\mathrm{H}$ & -5.21030 & -1.33750 & -18.21790 \\
\hline $\mathrm{H}$ & -2.20500 & -0.70300 & -18.07950 \\
\hline $\mathrm{H}$ & -4.36900 & -2.85780 & -19.73470 \\
\hline $\mathrm{O}$ & -3.41930 & -0.85330 & -14.35500 \\
\hline $\mathrm{H}$ & -2.08380 & -1.47190 & -15.76310 \\
\hline $\mathrm{H}$ & -3.49050 & 0.41780 & -18.49500 \\
\hline $\mathrm{C}$ & -4.05700 & -3.27800 & -10.65060 \\
\hline $\mathrm{H}$ & -4.27720 & -2.99630 & -11.68110 \\
\hline $\mathrm{H}$ & -5.00550 & -3.32890 & -10.11630 \\
\hline $\mathrm{C}$ & -3.34390 & -4.62920 & -10.63180 \\
\hline $\mathrm{H}$ & -3.05830 & -4.86300 & -9.60620 \\
\hline $\mathrm{H}$ & -2.40700 & -4.55590 & -11.18150 \\
\hline $\mathrm{C}$ & -5.83650 & -7.88840 & -12.07330 \\
\hline $\mathrm{C}$ & -5.13660 & -7.09410 & -13.00180 \\
\hline $\mathrm{C}$ & -4.29460 & -6.04240 & -12.56950 \\
\hline $\mathrm{C}$ & -4.20700 & -5.75670 & -11.17870 \\
\hline $\mathrm{C}$ & -4.93420 & -6.54050 & -10.25750 \\
\hline $\mathrm{C}$ & -5.73530 & -7.61000 & -10.69560 \\
\hline $\mathrm{H}$ & -5.20330 & -7.31860 & -14.05560 \\
\hline $\mathrm{H}$ & -4.85950 & -6.32540 & -9.20190 \\
\hline $\mathrm{C}$ & -2.16840 & -5.48380 & -13.77240 \\
\hline $\mathrm{C}$ & -3.50120 & -5.30350 & -13.58130 \\
\hline $\mathrm{C}$ & 0.39350 & -8.47380 & -12.02130 \\
\hline $\mathrm{C}$ & 0.74000 & -7.11320 & -11.87170 \\
\hline $\mathrm{C}$ & -0.11300 & -6.12980 & -12.42710 \\
\hline $\mathrm{C}$ & -1.27780 & -6.48220 & -13.14640 \\
\hline $\mathrm{C}$ & -1.58960 & -7.84950 & -13.29770 \\
\hline $\mathrm{C}$ & -0.76460 & -8.83930 & -12.73370 \\
\hline $\mathrm{H}$ & 0.11640 & -5.08990 & -12.28360 \\
\hline $\mathrm{C}$ & -4.25910 & -4.26610 & -14.34460 \\
\hline $\mathrm{O}$ & -3.48990 & -3.57310 & -15.21180 \\
\hline $\mathrm{O}$ & -5.47010 & -4.08150 & -14.22750 \\
\hline $\mathrm{C}$ & -1.11110 & 3.20070 & -12.67810 \\
\hline $\mathrm{O}$ & -0.03310 & 2.65890 & -12.92210 \\
\hline $\mathrm{H}$ & -2.44460 & 4.92370 & -12.83380 \\
\hline $\mathrm{C}$ & -0.47800 & 5.45620 & -13.41070 \\
\hline $\mathrm{C}$ & -1.42520 & 4.60090 & -12.98260 \\
\hline
\end{tabular}




$\begin{array}{rrrr}\mathrm{H} & 0.53480 & 5.09640 & -13.53660 \\ \mathrm{C} & -1.09600 & 9.63020 & -14.23660 \\ \mathrm{C} & 0.18700 & 9.15250 & -13.91370 \\ \mathrm{C} & 0.37860 & 7.77680 & -13.66840 \\ \mathrm{C} & -0.70580 & 6.87180 & -13.74150 \\ \mathrm{C} & -1.99210 & 7.36700 & -14.06040 \\ \mathrm{C} & -2.17610 & 8.73710 & -14.31290 \\ \mathrm{H} & 1.02850 & 9.82610 & -13.84210 \\ \mathrm{H} & 1.36440 & 7.41890 & -13.40630 \\ \mathrm{H} & -2.84520 & 6.70760 & -14.12690 \\ \mathrm{O} & -3.39320 & 9.25140 & -14.62920 \\ \mathrm{H} & -3.24760 & 10.18220 & -14.75270 \\ \mathrm{O} & -1.33380 & 10.95410 & -14.47230 \\ \mathrm{H} & -0.56550 & 11.49570 & -14.37430 \\ \mathrm{O} & -6.40030 & -8.38180 & -9.78640 \\ \mathrm{H} & -6.27790 & -8.10580 & -8.89360 \\ \mathrm{O} & -6.59170 & -8.94180 & -12.50230 \\ \mathrm{H} & -6.66710 & -9.01430 & -13.43880 \\ \mathrm{O} & 1.19050 & -9.44300 & -11.48430 \\ \mathrm{H} & 0.86850 & -10.32020 & -11.60740 \\ \mathrm{H} & -1.66270 & -4.87930 & -14.50980 \\ \mathrm{H} & -2.46380 & -8.15120 & -13.85510 \\ \mathrm{O} & -3.09460 & 0.03160 & -8.25350 \\ \mathrm{H} & -2.56950 & 0.70820 & -7.84270 \\ \mathrm{H} & -1.02350 & -9.87930 & -12.86340 \\ \mathrm{O} & 1.89960 & -6.81740 & -11.18410 \\ \mathrm{C} & 3.14390 & -7.04690 & -11.83170 \\ \mathrm{H} & 3.21550 & -8.06450 & -12.22000 \\ \mathrm{H} & 3.30520 & -6.33820 & -12.64470 \\ \mathrm{H} & 3.95340 & -6.91410 & -11.11360\end{array}$


Data for Macrocycle 15_conformer A

103

campione

C $\quad-3.05400$

$\begin{array}{llll}\text { C } & -2.15930 & -0.87160 & -10.63620\end{array}$

$\begin{array}{llll}\text { C } & -2.34000 & 1.52790 & -10.59550\end{array}$

$\begin{array}{llll}\text { C } & -2.15440 & 1.43240 & -12.11740\end{array}$

$\begin{array}{llll}\text { C } & -3.05450 & 0.32760 & -12.68340\end{array}$

$\begin{array}{llll}\text { O } & -2.70460 & -0.87940 & -12.01310\end{array}$

$\begin{array}{llll}\mathrm{O} & -1.29490 & 2.37040 & -10.10170\end{array}$

$\begin{array}{llll}\text { C } & -2.81070 & 0.08520 & -14.17960\end{array}$

$\mathrm{H} \quad-1.09630 \quad-0.16200 \quad-9.99260$

$\mathrm{H} \quad-3.35130 \quad 1.92760 \quad-10.36900$

$\mathrm{H} \quad-1.09230 \quad 1.20940 \quad-12.36500$

$\mathrm{H} \quad-4.12850 \quad 0.56870 \quad-12.51550$

$\begin{array}{llll}\mathrm{H} & -3.06770 & 0.99010 & -14.77040\end{array}$

$\mathrm{H} \quad-1.73970 \quad-0.15120 \quad-14.35950$

$\begin{array}{llll}\text { O } & -2.74910 & -2.17430 & -10.15390\end{array}$

$\begin{array}{llll}\mathrm{H} & -4.13070 & -0.63470 & -10.48950\end{array}$

$\begin{array}{llll}\text { C } & -3.36670 & -1.47040 & -15.90190\end{array}$

$\begin{array}{llll}\text { C } & -4.15090 & -2.77750 & -16.08780\end{array}$

$\begin{array}{llll}\text { C } & -4.05160 & -3.23970 & -17.54490\end{array}$

$\begin{array}{llll}\text { C } & -4.47610 & -2.10450 & -18.48160\end{array}$

$\begin{array}{llll}\text { C } & -3.66190 & -0.84900 & -18.16230\end{array}$

$\begin{array}{llll}\text { O } & -3.85390 & -0.48360 & -16.80250\end{array}$

$\begin{array}{llll}\mathrm{O} & -4.98710 & -4.30790 & -17.71560\end{array}$

$\begin{array}{llll}\text { O } & -4.13990 & -2.49200 & -19.81630\end{array}$

$\mathrm{H} \quad-5.21670 \quad-2.62410 \quad-15.80980$

$\mathrm{H} \quad-3.00870 \quad-3.54750 \quad-17.77110$

$\begin{array}{llll}\mathrm{H} & -5.55830 & -1.88430 & -18.36330\end{array}$

$\begin{array}{llll}\mathrm{H} & -2.57970 & -1.00610 & -18.36110\end{array}$

$\begin{array}{llll}\mathrm{O} & -3.62880 & -1.00790 & -14.58470\end{array}$

$\mathrm{H} \quad-2.27820 \quad-1.64310 \quad-16.05180$

$\begin{array}{llll}\mathrm{H} & -3.99130 & -0.00610 & -18.80730\end{array}$

C $\quad-3.65620 \quad-3.14620 \quad-10.67350$

$\begin{array}{llll}\mathrm{H} & -3.82060 & -2.98410 & -11.75920\end{array}$

$\begin{array}{llll}\mathrm{H} & -4.64020 & -3.03730 & -10.16740\end{array}$

$\begin{array}{llll}\text { C } & -3.09670 & -4.55100 & -10.44680\end{array}$

$\mathrm{H} \quad-2.87730 \quad-4.68940 \quad-9.36360$

$\begin{array}{llll}\mathrm{H} & -2.11830 & -4.65320 & -10.96410\end{array}$

$\begin{array}{llll}\text { C } & -5.92910 & -7.58520 & -11.67620\end{array}$

$\begin{array}{llll}\text { C } & -5.15700 & -6.93140 & -12.63810\end{array}$

$\begin{array}{llll}\text { C } & -4.21340 & -5.96160 & -12.27680\end{array}$

$\begin{array}{llll}\text { C } & -4.05720 & -5.62290 & -10.91840\end{array}$

$\begin{array}{llll}\text { C } & -4.83740 & -6.28570 & -9.95950\end{array}$

$\begin{array}{llll}\text { C } & -5.76500 & -7.26310 & -10.32770\end{array}$

$\mathrm{H} \quad-5.28600 \quad-7.18250 \quad-13.70410$

$\begin{array}{llll}\mathrm{H} & -4.72120 & -6.02610 & -8.89420\end{array}$

$\begin{array}{llll}\text { C } & -2.11640 & -5.51900 & -13.54720\end{array}$

$\begin{array}{llll}\text { C } & -3.43010 & -5.31160 & -13.34290\end{array}$

$\begin{array}{llll}\text { C } & 0.38090 & -8.25970 & -11.37650\end{array}$

$\begin{array}{llll}\text { C } & -0.08230 & -8.58080 & -12.66280\end{array}$

$\begin{array}{llll}\text { C } & -0.89490 & -7.66470 & -13.34020\end{array}$

$\begin{array}{llll}\text { C } & -1.25220 & -6.43470 & -12.77870\end{array}$

$\begin{array}{llll}\text { C } & -0.79500 & -6.13720 & -11.49270\end{array}$

$\begin{array}{llll}\text { C } & 0.01490 & -7.03780 & -10.80130\end{array}$

$\begin{array}{llll}\mathrm{H} & -1.25260 & -7.92160 & -14.35110\end{array}$

$\begin{array}{llll}\text { C } & -4.13780 & -4.36120 & -14.24450\end{array}$

$\begin{array}{llll}\text { O } & -3.50060 & -3.77700 & -15.28850\end{array}$

$\begin{array}{llll}\text { O } & -5.30140 & -4.09520 & -14.04210\end{array}$

$\begin{array}{llll}\text { O } & -6.52120 & -7.90450 & -9.39360\end{array}$

$\mathrm{H} \quad-6.34510 \quad-7.61850 \quad-8.48130$

$\begin{array}{llll}\mathrm{O} & -6.84130 & -8.53280 & -12.03010\end{array}$

$\begin{array}{llll}\mathrm{H} & -6.90070 & -8.68180 & -12.98900\end{array}$

$\begin{array}{llll}\mathrm{O} & 1.17460 & -9.11250 & -10.67140\end{array}$

$\begin{array}{llll}\mathrm{H} & 1.43530 & -8.78420 & -9.79400\end{array}$

$\begin{array}{llll}\mathrm{H} & -1.61330 & -4.93240 & -14.33440\end{array}$

$\begin{array}{llll}\mathrm{H} & -1.07830 & -5.19300 & -11.00240\end{array}$

$\begin{array}{llll}\text { O } & -2.62530 & 0.28430 & -8.58620\end{array}$ 


$\begin{array}{lrrr}\mathrm{H} & 0.36280 & -6.77330 & -9.78860 \\ \mathrm{O} & 0.18060 & -9.73270 & -13.35750 \\ \mathrm{C} & 0.98080 & -10.77700 & -12.82580 \\ \mathrm{H} & 2.01330 & -10.42100 & -12.62690 \\ \mathrm{H} & 0.52610 & -11.19130 & -11.90160 \\ \mathrm{H} & 1.04270 & -11.59760 & -13.57060 \\ \mathrm{O} & -2.59000 & 2.66760 & -12.69120 \\ \mathrm{C} & -1.48080 & 3.48240 & -9.35560 \\ \mathrm{O} & -0.49940 & 4.10630 & -9.02310 \\ \mathrm{C} & -1.89280 & -0.00560 & -7.48760 \\ \mathrm{O} & -2.40470 & 0.17120 & -6.40590 \\ \mathrm{C} & -1.68420 & 3.56950 & -13.11040 \\ \mathrm{O} & -1.96830 & 4.60230 & -13.66480 \\ \mathrm{C} & -5.03770 & -2.75150 & -20.79370 \\ \mathrm{O} & -4.61110 & -3.06800 & -21.88050 \\ \mathrm{C} & -4.67920 & -5.53660 & -18.18900 \\ \mathrm{O} & -5.57320 & -6.34610 & -18.28240 \\ \mathrm{C} & -2.87520 & 3.92860 & -8.95090 \\ \mathrm{H} & -3.49230 & 4.17430 & -9.84310 \\ \mathrm{H} & -3.39450 & 3.14230 & -8.36010 \\ \mathrm{H} & -2.82930 & 4.84230 & -8.31790 \\ \mathrm{C} & -0.47490 & -0.53580 & -7.61290 \\ \mathrm{H} & -0.44980 & -1.48660 & -8.18960 \\ \mathrm{H} & 0.18800 & 0.20530 & -8.11140 \\ \mathrm{H} & -0.04190 & -0.74550 & -6.60980 \\ \mathrm{C} & -6.53070 & -2.65080 & -20.53100 \\ \mathrm{H} & -6.82240 & -1.61000 & -20.26860 \\ \mathrm{H} & -7.11070 & -2.93930 & -21.43550 \\ \mathrm{H} & -6.84310 & -3.32990 & -19.70780 \\ \mathrm{C} & -3.25690 & -5.88440 & -18.59230 \\ \mathrm{H} & -2.56480 & -5.81180 & -17.72440 \\ \mathrm{H} & -3.19900 & -6.92720 & -18.97570 \\ \mathrm{H} & -2.89170 & -5.21380 & -19.40100 \\ \mathrm{C} & -0.24230 & 3.18750 & -12.80800 \\ \mathrm{H} & 0.44270 & 3.98660 & -13.18750 \\ \mathrm{H} & -0.10980 & 3.07670 & -11.70250 \\ \mathrm{H} & 0.00020 & 2.21820 & -13.31160\end{array}$


Data for Macrocycle 15_conformer B

103

campione

\begin{tabular}{|c|c|c|c|}
\hline $\mathrm{C}$ & 0.75050 & -5.02420 & -18.00770 \\
\hline $\mathrm{C}$ & 1.99260 & -4.23210 & -18.36310 \\
\hline $\mathrm{C}$ & 2.54610 & -4.54060 & -19.76480 \\
\hline $\mathrm{C}$ & 2.64780 & -6.04770 & -19.98980 \\
\hline $\mathrm{C}$ & 1.26630 & -6.58230 & -19.68330 \\
\hline $\mathrm{O}$ & 0.97570 & -6.40890 & -18.28080 \\
\hline $\mathrm{O}$ & 3.91830 & -3.98330 & -19.83700 \\
\hline $\mathrm{C}$ & 1.03880 & -8.21540 & -20.05580 \\
\hline $\mathrm{H}$ & 2.83390 & -4.43210 & -17.66570 \\
\hline $\mathrm{H}$ & 1.94450 & -4.15480 & -20.61580 \\
\hline $\mathrm{H}$ & 3.47500 & -6.47220 & -19.38340 \\
\hline $\mathrm{H}$ & 0.52440 & -6.04130 & -20.31500 \\
\hline $\mathrm{H}$ & 1.80440 & -8.40640 & -20.83660 \\
\hline $\mathrm{H}$ & 1.35790 & -8.80750 & -19.17170 \\
\hline $\mathrm{O}$ & 0.49890 & -4.78590 & -16.63130 \\
\hline $\mathrm{H}$ & -0.10440 & -4.71310 & -18.63450 \\
\hline $\mathrm{C}$ & -0.83590 & -9.76950 & -20.08950 \\
\hline $\mathrm{C}$ & -2.07470 & -9.52380 & -19.09200 \\
\hline $\mathrm{C}$ & -2.90560 & -10.80710 & -18.91580 \\
\hline $\mathrm{C}$ & -3.32450 & -11.17860 & -20.32400 \\
\hline $\mathrm{C}$ & -2.02650 & -11.44680 & -21.17720 \\
\hline $\mathrm{O}$ & -1.45000 & -10.19510 & -21.31760 \\
\hline $\mathrm{O}$ & -4.09980 & -10.41190 & -18.29810 \\
\hline $\mathrm{O}$ & -3.87840 & -12.48810 & -20.22250 \\
\hline $\mathrm{H}$ & -2.77410 & -8.76830 & -19.51450 \\
\hline $\mathrm{H}$ & -2.25070 & -11.54210 & -18.40150 \\
\hline $\mathrm{H}$ & -3.94720 & -10.45580 & -20.89260 \\
\hline $\mathrm{H}$ & -1.32930 & -12.16960 & -20.70060 \\
\hline $\mathrm{O}$ & -0.26320 & -8.44520 & -20.43820 \\
\hline $\mathrm{H}$ & -0.03370 & -10.41590 & -19.67010 \\
\hline $\mathrm{H}$ & -2.31970 & -11.87170 & -22.16130 \\
\hline $\mathrm{C}$ & -0.83880 & -4.32620 & -16.55860 \\
\hline $\mathrm{H}$ & -1.50750 & -4.85370 & -17.27060 \\
\hline $\mathrm{H}$ & -0.84140 & -3.24960 & -16.83720 \\
\hline $\mathrm{C}$ & -1.41980 & -4.63510 & -15.18190 \\
\hline $\mathrm{H}$ & -1.61440 & -3.61650 & -14.77550 \\
\hline $\mathrm{H}$ & -0.65870 & -5.09430 & -14.51970 \\
\hline $\mathrm{C}$ & -5.00580 & -6.98340 & -14.97980 \\
\hline $\mathrm{C}$ & -3.79770 & -7.52800 & -15.41100 \\
\hline $\mathrm{C}$ & -2.67530 & -6.73760 & -15.51440 \\
\hline $\mathrm{C}$ & -2.66630 & -5.43280 & -15.13260 \\
\hline $\mathrm{C}$ & -3.84740 & -4.95080 & -14.61100 \\
\hline $\mathrm{C}$ & -5.05660 & -5.66090 & -14.61560 \\
\hline $\mathrm{H}$ & -3.65050 & -8.55460 & -15.80320 \\
\hline $\mathrm{H}$ & -3.88960 & -3.92600 & -14.20670 \\
\hline $\mathrm{C}$ & -0.33930 & -7.50090 & -15.58720 \\
\hline $\mathrm{C}$ & -1.52860 & -7.29850 & -16.17970 \\
\hline $\mathrm{C}$ & 1.31040 & -6.90290 & -11.62790 \\
\hline $\mathrm{C}$ & -0.04180 & -6.91380 & -11.76830 \\
\hline $\mathrm{C}$ & -0.59850 & -7.09520 & -13.03780 \\
\hline $\mathrm{C}$ & 0.16990 & -7.27250 & -14.16730 \\
\hline $\mathrm{C}$ & 1.59510 & -7.37080 & -13.95610 \\
\hline $\mathrm{C}$ & 2.16370 & -7.08100 & -12.67230 \\
\hline $\mathrm{H}$ & -1.67970 & -7.17100 & -13.23590 \\
\hline $\mathrm{C}$ & -1.71140 & -7.72800 & -17.57010 \\
\hline $\mathrm{O}$ & -1.46750 & -9.02520 & -17.87990 \\
\hline $\mathrm{O}$ & -2.14690 & -6.92780 & -18.41770 \\
\hline $\mathrm{O}$ & -6.19200 & -5.05460 & -14.04550 \\
\hline $\mathrm{H}$ & -6.20920 & -4.16160 & -13.66130 \\
\hline $\mathrm{O}$ & -6.12800 & -7.73330 & -14.90450 \\
\hline $\mathrm{H}$ & -6.10660 & -8.46610 & -15.54300 \\
\hline $\mathrm{O}$ & 1.81990 & -6.82000 & -10.38750 \\
\hline $\mathrm{H}$ & 2.73710 & -7.13770 & -10.33880 \\
\hline $\mathrm{H}$ & 0.44550 & -7.77720 & -16.31300 \\
\hline $\mathrm{H}$ & 2.26450 & -7.43230 & -14.83030 \\
\hline $\mathrm{O}$ & 1.76880 & -2.84340 & -18.35650 \\
\hline
\end{tabular}




$\begin{array}{lrll}\mathrm{H} & 3.25620 & -6.99850 & -12.54850 \\ \mathrm{O} & -0.91940 & -6.78360 & -10.64900 \\ \mathrm{C} & -2.33160 & -6.49550 & -10.73700 \\ \mathrm{H} & -2.92250 & -7.41590 & -10.93180 \\ \mathrm{H} & -2.64410 & -6.20610 & -9.71190 \\ \mathrm{H} & -2.53740 & -5.65990 & -11.43790 \\ \mathrm{O} & 2.91800 & -6.32160 & -21.36420 \\ \mathrm{C} & 4.40920 & -3.11320 & -20.75140 \\ \mathrm{O} & 5.56820 & -2.78480 & -20.56630 \\ \mathrm{C} & 2.24240 & -1.73660 & -17.61750 \\ \mathrm{O} & 1.68410 & -0.68490 & -17.72730 \\ \mathrm{C} & 4.14760 & -6.51530 & -21.96170 \\ \mathrm{O} & 4.15520 & -6.57950 & -23.14300 \\ \mathrm{C} & -5.11320 & -12.83860 & -20.70540 \\ \mathrm{O} & -5.64250 & -13.85140 & -20.34570 \\ \mathrm{C} & -4.71420 & -10.95290 & -17.24320 \\ \mathrm{O} & -5.63670 & -10.34410 & -16.75500 \\ \mathrm{C} & 3.70850 & -2.68340 & -22.04130 \\ \mathrm{H} & 4.19650 & -1.72160 & -22.31260 \\ \mathrm{H} & 4.01740 & -3.40060 & -22.83350 \\ \mathrm{H} & 2.60070 & -2.60150 & -21.97900 \\ \mathrm{C} & 3.38760 & -1.97110 & -16.62840 \\ \mathrm{H} & 3.51320 & -1.10310 & -15.94450 \\ \mathrm{H} & 3.12830 & -2.87890 & -16.04110 \\ \mathrm{H} & 4.30110 & -2.20970 & -17.21680 \\ \mathrm{C} & -5.89990 & -11.90220 & -21.52910 \\ \mathrm{H} & -6.82380 & -12.31040 & -21.99510 \\ \mathrm{H} & -6.20330 & -11.05590 & -20.87390 \\ \mathrm{H} & -5.23720 & -11.58380 & -22.36380 \\ \mathrm{C} & -4.29600 & -12.26370 & -16.64770 \\ \mathrm{H} & -5.11700 & -12.65380 & -16.00660 \\ \mathrm{H} & -4.08990 & -13.00900 & -17.44750 \\ \mathrm{H} & -3.42580 & -11.97320 & -16.01970 \\ \mathrm{C} & 5.37520 & -6.65530 & -21.03210 \\ \mathrm{H} & 5.40070 & -5.80750 & -20.31210 \\ \mathrm{H} & 5.39430 & -7.57700 & -20.41050 \\ \mathrm{H} & 6.34620 & -6.52150 & -21.55770\end{array}$

